\title{
New steroidal derivatives synthesized using $3 \beta$-hydroxyandrosten-17-one as starting material
}

\author{
NEHAD A. ABD EL-LATIF ${ }^{1}$ \\ MOHAMED M. ABDULLA 2 \\ ABD EL-GALIL E. AMR ${ }^{3}$ \\ ${ }^{1}$ Natural Compounds Department \\ National Research Center, Cairo \\ Dokki, Egypt \\ ${ }^{2}$ Research Units, Hi-Care \\ Pharmaceutical Co., Cairo, Egypt \\ 3 Applied Organic Chemistry \\ Department National Research Center \\ Cairo, Dokki, Egypt
}

\begin{abstract}
In this study, we synthesized some new substituted steroidal derivatives using 3 $\beta$-hydroxyandrosten-17-one (dehydroepiandrosterone) as starting material. The synthesized steroidal derivatives 1-11 were evaluated for their androgenic-anabolic activities compared to testosterone as positive control. Details of the synthesis, spectroscopic data and toxicity $\left(L D_{50}\right)$ of synthesized compounds are reported.
\end{abstract}

Keywords: dehydroepiandrosterone, steroidal derivatives, androgenic-anabolic agents

Accepted February 5, 2008

Natural steroids and their synthetic congeners were extensively studied during the last decade $(1,2)$. In a previous work, some new heterocyclic compounds showing antiparkinsonian (3), antitumor (4-6), antimicrobial (7-10) and anti-inflammatory (11) activity were synthesized. We found that certain substituted steroidal derivatives showed androgenic, anabolic and anti-inflammatory activities (12). Steroidal pyrazolines are an interesting group of compounds, many of which possess wide spread pharmacological properties such as analgesic, antipyretic and antiandrogenic activities $(13,14)$. These derivatives are also well known for their pronounced anti-inflammatory (15) activity and are used as potent antidiabetic agents (16). In addition, the pharmacological and antitumor activities of many heterocyclic compounds have been reviewed (17-19). Recently, nitrogenous derivatives exhibited a general ionophoric potency for divalent cations (20) and are used as novel thiocyanate-selective membrane sensors (21). In view of these reports and in continuation of our previous work in heterocyclic chemistry, we have herein synthesized some new compounds containing steroidal structure for the evaluation of androgenic-anabolic activity as compared to testosterone as standard drug (Fig. $1)$.

\footnotetext{
*Correspondence, e-mail: aamr1963@yahoo.com
} 


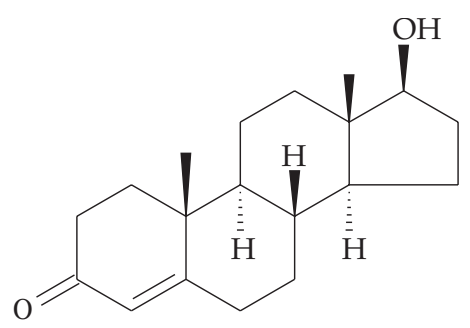

Fig. 1. Testosterone structural formula.

EXPERIMENTAL

Melting points were determined on an Electrothermal IA 9000 apparatus (Electrothermal, UK) and are uncorrected. Elemental analyses for the final compounds were performed on Elementar, Vario EL (USA) at the Microanalytical Unit, National Research Centre, Cairo Egypt, and were found within $\pm 0.4 \%$ of the theoretical values. The IR spectra (KBr) were recorded on an FT IR-8201 PC Spectrophotometer (Shimadzu, Japan). The ${ }^{1} \mathrm{H}$ NMR spectra were measured with Jeol $270 \mathrm{MHz}$ (Japan) in DMSO- $\mathrm{d}_{6}$ and the chemical shifts were recorded in $(\delta, \mathrm{ppm})$ relative to TMS. Mass spectra were run at 70 eV with a Finnigan SSQ 7000 (thermo-instrument system incorporation, USA) spectrometer using EI. The reactions were followed by TLC (silica gel, aluminum sheets $60 \mathrm{~F}_{254}$, Merck, Germany). Testosterone and dehydroepiandrosterone were purchased from Aldrich (Germany).

Physicochemical and spectral data for the synthesized compounds are given in Tables I and II, respectively.

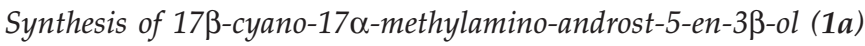

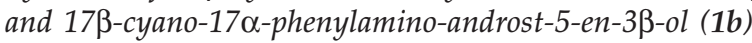

To a stirred solution of dehydroepiandrosterone $(0.29 \mathrm{~g}, 1 \mathrm{mmol})$ in absolute ethanol $(25 \mathrm{~mL})$, the appropriate primary amine, namely, methylamine or aniline $(1 \mathrm{mmol})$ in ethanol $(5 \mathrm{~mL})$ was added dropwise. Hydrochloric acid $(5 \mathrm{~mL}, 10 \%)$ was added to the reaction mixture; then, potassium cyanide $(0.1 \mathrm{~g}, \sim 1 \mathrm{mmol})$ in water $(10 \mathrm{~mL})$ was added dropwise. The reaction mixture was stirred at room temperature for 96 hours, diluted with water $(100 \mathrm{~mL})$ and neutralized with sodium bicarbonate $(10 \%)$. The resulting mixture was extracted with chloroform, washed with water, dried over anhydrous sodium sulphate and evaporated under reduced pressure. The obtained residue was solidified with diethyl ether, the obtained solid was filtered off, dried and crystallized from the proper solvent to give the corresponding carbonitriles $\mathbf{1} \mathbf{a}, \mathbf{b}$, respectively (Scheme 1).

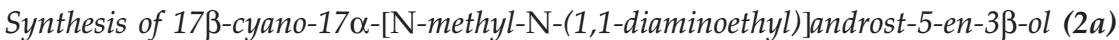

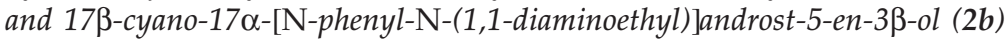

A mixture of 1a,b (1 mmol) and dimethylamino-2-chloroethane $(0.17 \mathrm{~g}, 1.2 \mathrm{mmol})$ was refluxed for 7 hours in the presence of triethylamine $(0.2 \mathrm{~mL})$ as a catalyst in benzene. The reaction mixture was washed with water, $10 \%$ sodium bicarbonate, then with 
N. A. Abd El-Latif et al.: New steroidal derivatives synthesized using 33-hydroxyandrosten-17-one as starting material, Acta Pharm. 58 (2008) $43-59$.

Table I. Physical and analytical data of newly synthesized compounds

\begin{tabular}{|c|c|c|c|c|c|c|}
\hline $\begin{array}{l}\text { Compd. } \\
\text { No. }\end{array}$ & $\mathrm{R}$ & $\begin{array}{c}\text { Yield } \\
(\%)\end{array}$ & $\begin{array}{l}\text { M.p. } \\
\left({ }^{\circ} \mathrm{C}\right)\end{array}$ & $\begin{array}{c}{[\alpha]_{\mathrm{D}}^{25}} \\
(c 1, \mathrm{MeOH})\end{array}$ & $\begin{array}{c}\text { Color } \\
\text { (solvent for crystallization) }\end{array}$ & $\begin{array}{l}\text { Mol. formula } \\
\qquad\left(M_{\mathrm{r}}\right)^{\mathrm{a}}\end{array}$ \\
\hline 1a & $\mathrm{CH}_{3}$ & 98 & 281 & +91 & White powder $(\mathrm{MeOH})$ & $\mathrm{C}_{21} \mathrm{H}_{32} \mathrm{~N}_{2} \mathrm{O}(328.49)$ \\
\hline $1 b$ & $\mathrm{C}_{6} \mathrm{H}_{5}$ & 84 & 316 & +76 & Pale yellow powder (AcOEt) & $\mathrm{C}_{26} \mathrm{H}_{34} \mathrm{~N}_{2} \mathrm{O}(390.56)$ \\
\hline $2 a$ & $\mathrm{CH}_{3}$ & 81 & 217 & +79 & White powder (AcOMe) & $\mathrm{C}_{25} \mathrm{H}_{41} \mathrm{~N}_{3} \mathrm{O}$ (399.62) \\
\hline $2 b$ & $\mathrm{C}_{6} \mathrm{H}_{5}$ & 76 & 271 & +94 & White powder $(\mathrm{MeOH})$ & $\mathrm{C}_{30} \mathrm{H}_{43} \mathrm{~N}_{3} \mathrm{O}(461.69)$ \\
\hline $3 a$ & $\mathrm{CH}_{3}$ & 68 & 235 & +88 & White powder (EtOH) & $\mathrm{C}_{27} \mathrm{H}_{43} \mathrm{~N}_{3} \mathrm{O}(425.65)$ \\
\hline $3 b$ & $\mathrm{C}_{6} \mathrm{H}_{5}$ & 77 & 286 & +96 & White powder (EtOH) & $\mathrm{C}_{32} \mathrm{H}_{45} \mathrm{~N}_{3} \mathrm{O}(487.72)$ \\
\hline $4 a$ & $\mathrm{CH}_{3}$ & 64 & 196 & +66 & White powder $(\mathrm{AcOH})$ & $\mathrm{C}_{25} \mathrm{H}_{36} \mathrm{~N}_{2} \mathrm{O}_{3}(412.57)$ \\
\hline $4 b$ & $\mathrm{C}_{6} \mathrm{H}_{5}$ & 72 & 216 & +86 & White powder $(\mathrm{AcOH})$ & $\mathrm{C}_{30} \mathrm{H}_{38} \mathrm{~N}_{2} \mathrm{O}_{3}(474.64)$ \\
\hline $5 a$ & $\mathrm{CH}_{3}$ & 65 & 288 & +69 & White powder $(\mathrm{MeOH})$ & $\mathrm{C}_{25} \mathrm{H}_{30} \mathrm{~F}_{6} \mathrm{~N}_{2} \mathrm{O}_{3}(520.51)$ \\
\hline $5 b$ & $\mathrm{C}_{6} \mathrm{H}_{5}$ & 68 & 249 & +79 & Pale yellow powder $(\mathrm{MeOH})$ & $\mathrm{C}_{30} \mathrm{H}_{32} \mathrm{~F}_{6} \mathrm{~N}_{2} \mathrm{O}_{3}(582.58)$ \\
\hline $6 a$ & $\mathrm{CH}_{3}$ & 55 & 211 & +92 & White $(\mathrm{MeOH})$ & $\mathrm{C}_{21} \mathrm{H}_{38} \mathrm{~N}_{2} \mathrm{O}(334.54)$ \\
\hline $6 b$ & $\mathrm{C}_{6} \mathrm{H}_{5}$ & 65 & 218 & +76 & White powder (AcOEt) & $\mathrm{C}_{26} \mathrm{H}_{40} \mathrm{~N}_{2} \mathrm{O}$ (396.61) \\
\hline $7 a$ & $\mathrm{CH}_{3}$ & 67 & 199 & +96 & White (AcOMe) & $\mathrm{C}_{21} \mathrm{H}_{36} \mathrm{~N}_{2} \mathrm{O}(332.52)$ \\
\hline $7 \mathrm{~b}$ & $\mathrm{C}_{6} \mathrm{H}_{5}$ & 72 & 148 & +79 & White powder $(\mathrm{MeOH})$ & $\mathrm{C}_{26} \mathrm{H}_{38} \mathrm{~N}_{2} \mathrm{O}(394.60)$ \\
\hline $8 a$ & $\mathrm{CH}_{3}$ & 55 & 188 & +89 & White powder $(\mathrm{EtOH})$ & $\mathrm{C}_{21} \mathrm{H}_{34} \mathrm{~N}_{2} \mathrm{O}_{2}(346.51)$ \\
\hline $8 b$ & $\mathrm{C}_{6} \mathrm{H}_{5}$ & 49 & 198 & +77 & Orange powder (EtOH) & $\mathrm{C}_{26} \mathrm{H}_{36} \mathrm{~N}_{2} \mathrm{O}_{2}(408.58)$ \\
\hline $9 a$ & $\mathrm{CH}_{3}$ & 60 & 252 & +95 & White powder $(\mathrm{AcOH})$ & $\mathrm{C}_{21} \mathrm{H}_{33} \mathrm{NO}_{3}(347.49)$ \\
\hline $9 b$ & $\mathrm{C}_{6} \mathrm{H}_{5}$ & 62 & 249 & +75 & White powder $(\mathrm{AcOH})$ & $\mathrm{C}_{26} \mathrm{H}_{35} \mathrm{NO}_{3}(409.56)$ \\
\hline $10 a$ & $\mathrm{CH}_{3}$ & 69 & 207 & +93 & White powder $(\mathrm{MeOH})$ & $\mathrm{C}_{21} \mathrm{H}_{31} \mathrm{NO}_{3}(345.48)$ \\
\hline $10 \mathrm{~b}$ & $\mathrm{C}_{6} \mathrm{H}_{5}$ & 78 & 187 & +76 & White powder $(\mathrm{MeOH})$ & $\mathrm{C}_{26} \mathrm{H}_{33} \mathrm{NO}_{3}(407.55)$ \\
\hline $11 a$ & $\mathrm{CH}_{3}$ & 80 & 178 & +88 & Pale yellow powder $(\mathrm{AcOH})$ & $\mathrm{C}_{23} \mathrm{H}_{37} \mathrm{~N}_{3} \mathrm{O}(371.56)$ \\
\hline $11 \mathrm{~b}$ & $\mathrm{C}_{6} \mathrm{H}_{5}$ & 76 & 213 & +79 & White powder $(\mathrm{MeOH})$ & $\mathrm{C}_{28} \mathrm{H}_{39} \mathrm{~N}_{3} \mathrm{O}(433.63)$ \\
\hline
\end{tabular}

a Confirmed by elemental analysis showing values within $\pm 0.4 \%$ of the theoretical values unless otherwise stated.

water and dried over anhydrous sodium sulphate. The benzene part was evaporated under reduced pressure and the residue obtained was solidified with $n$-hexane. The obtained solid was filtered off, dried and crystallized from the proper solvent to give the derivatives $\mathbf{2} \mathbf{a}, \mathbf{b}$, respectively (Scheme 1 ).

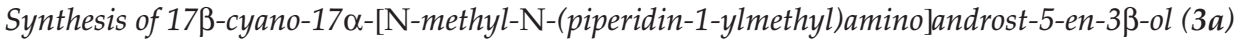

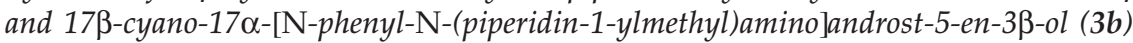

A mixture of carbonitrile derivatives $\mathbf{1 a}, \mathbf{b}(1 \mathrm{mmol})$ and piperidine $(4 \mathrm{mmol})$ was refluxed for 2 hours in the presence of powdered paraformaldehyde $(12 \mathrm{mg})$ in $1 \%$ hydrochloric acid in ethanol $(15 \mathrm{~mL})$. The reaction mixture was concentrated under reduced pressure to dryness; the solid formed was collected by filtration, washed with ethanol, dried and crystallized from the proper solvent to afford the corresponding piperidenyl derivatives $\mathbf{3 a}, \mathbf{b}$, respectively (Scheme 1 ). 
N. A. Abd El-Latif et al.: New steroidal derivatives synthesized using 3ß-hydroxyandrosten-17-one as starting material, Acta Pharm. 58 (2008) $43-59$.

Table II. Spectral data of newly synthesized compounds

\begin{tabular}{|c|c|c|c|}
\hline $\begin{array}{c}\text { Compd. } \\
\text { No. }\end{array}$ & $\begin{array}{l}\text { Mass } \\
(m / z, \%)\end{array}$ & $\begin{array}{l}\text { IR } \\
\left(v, \mathrm{~cm}^{-1}\right)\end{array}$ & $\begin{array}{l}{ }^{1} \mathrm{H} \text { NMR } \\
(\delta, \mathrm{ppm})\end{array}$ \\
\hline 1a & $\begin{array}{l}328.49\left(\mathrm{M}^{+}\right] \\
(32), 254 \\
(100)\end{array}$ & $\begin{array}{l}3448-3378 \\
(\mathrm{OH}, \mathrm{NH}) \\
2224(\mathrm{C} \equiv \mathrm{N}), \\
1634(\mathrm{C}=\mathrm{C})\end{array}$ & $\begin{array}{l}0.76\left(\mathrm{~s}, 3 \mathrm{H}, \mathrm{CH}_{3}, \mathrm{C}-19\right), 0.88\left(\mathrm{~s}, 3 \mathrm{H}, \mathrm{CH}_{3}, \mathrm{C}-18\right), 0.96-1.04 \\
(\mathrm{~m}, 1 \mathrm{H}, \mathrm{CH}), 1.15-1.25\left(\mathrm{~m}, 4 \mathrm{H}, 2 \mathrm{CH}_{2}\right), 1.42-1.56(\mathrm{~m}, 6 \mathrm{H}, \\
\left.3 \mathrm{CH}_{2}\right), 1.60-1.80\left(\mathrm{~m}, 4 \mathrm{H}, 2 \mathrm{CH}_{2}\right), 1.94-1.98(\mathrm{~m}, 1 \mathrm{H}, \mathrm{CH}) \\
2.38\left(\mathrm{~s}, 3 \mathrm{H}, \mathrm{N}-\mathrm{CH}_{3}\right), 2.42-2.48\left(\mathrm{~m}, 2 \mathrm{H}, \mathrm{CH}_{2}\right), 2.56-2.59(\mathrm{~m}, \\
1 \mathrm{H}, \mathrm{CH}), 3.55-3.60(\mathrm{~m}, 1 \mathrm{H}, 3 \alpha-\mathrm{CH}), 3.92(\mathrm{~s}, 1 \mathrm{H}, \mathrm{NH}, \mathrm{ex}- \\
\left.\text { changeable with } \mathrm{D}_{2} \mathrm{O}\right), 5.58-5.64(\mathrm{~m}, 1 \mathrm{H}, \mathrm{CH}, \mathrm{C}-6), 10.00 \\
\left(\mathrm{~s}, 1 \mathrm{H}, \mathrm{OH}, \text { exchangeable with } \mathrm{D}_{2} \mathrm{O}\right)\end{array}$ \\
\hline $1 b$ & $\begin{array}{l}390\left[\mathrm{M}^{+}\right] \\
(12), 274 \\
(100)\end{array}$ & $\begin{array}{l}3448-3378 \\
\text { (broad band, } \\
\text { OH, NH), } \\
2224(\mathrm{C} \equiv \mathrm{N}), \\
1634(\mathrm{C}=\mathrm{C})\end{array}$ & $\begin{array}{l}0.78\left(\mathrm{~s}, 3 \mathrm{H}, \mathrm{CH}_{3}, \mathrm{C}-19\right), 0.87\left(\mathrm{~s}, 3 \mathrm{H}, \mathrm{CH}_{3}, \mathrm{C}-18\right), 0.95-1.05 \\
(\mathrm{~m}, 1 \mathrm{H}, \mathrm{CH}), 1.10-1.24\left(\mathrm{~m}, 4 \mathrm{H}, 2 \mathrm{CH}_{2}\right), 1.43-1.55(\mathrm{~m}, 6 \mathrm{H}, \\
\left.3 \mathrm{CH}_{2}\right), 1.62-1.79\left(\mathrm{~m}, 4 \mathrm{H}, 2 \mathrm{CH}_{2}\right), 1.97-2.04(\mathrm{~m}, 1 \mathrm{H}, \mathrm{CH}) \\
2.44-2.50\left(\mathrm{~m}, 2 \mathrm{H}, \mathrm{CH}_{2}\right), 2.58-2.62(\mathrm{~m}, 1 \mathrm{H}, \mathrm{CH}), 3.56-3.64 \\
(\mathrm{~m}, 1 \mathrm{H}, 3 \alpha-\mathrm{CH}), 4.05(\mathrm{~s}, 1 \mathrm{H}, \mathrm{NH}, \text { exchangeable with } \\
\left.\mathrm{D}_{2} \mathrm{O}\right), 5.55-5.65(\mathrm{~m}, 1 \mathrm{H}, \mathrm{CH}, \mathrm{C}-6), 7.23-7.50(\mathrm{~m}, 5 \mathrm{H}, \\
\text { Ar-H), } 10.05\left(\mathrm{~s}, 1 \mathrm{H}, \mathrm{OH}, \text { exchangeable with } \mathrm{D}_{2} \mathrm{O}\right)\end{array}$ \\
\hline $2 a$ & $\begin{array}{l}399\left[\mathrm{M}^{+}\right] \\
(22), 301 \\
(100)\end{array}$ & $\begin{array}{l}3451-3391 \\
(\mathrm{OH}), 2227 \\
(\mathrm{C} \equiv \mathrm{N}), 1632 \\
(\mathrm{C}=\mathrm{C})\end{array}$ & $\begin{array}{l}0.78\left(\mathrm{~s}, 3 \mathrm{H}, \mathrm{CH}_{3}, \mathrm{C}-19\right), 0.94\left(\mathrm{~s}, 3 \mathrm{H}, \mathrm{CH}_{3}, \mathrm{C}-18\right), 0.96-1.00 \\
(\mathrm{~m}, 1 \mathrm{H}, \mathrm{CH}), 1.10-1.23\left(\mathrm{~m}, 4 \mathrm{H}, 2 \mathrm{CH}_{2}\right), 1.38-1.55(\mathrm{~m}, 6 \mathrm{H}, \\
\left.3 \mathrm{CH}_{2}\right), 1.62-1.79\left(\mathrm{~m}, 4 \mathrm{H}, 2 \mathrm{CH}_{2}\right), 1.82-1.85\left(\mathrm{~m}, 4 \mathrm{H}, 2 \mathrm{CH}_{2}\right), \\
1.97-2.00(\mathrm{~m}, 1 \mathrm{H}, \mathrm{CH}), 2.32,2.36,2.39(3 \mathrm{~s}, 9 \mathrm{H}, 3 \times \\
\left.\mathrm{N}-\mathrm{CH}_{3}\right), 2.44-2.48\left(\mathrm{~m}, 2 \mathrm{H}, \mathrm{CH}_{2}\right), 2.55-2.60(\mathrm{~m}, 1 \mathrm{H}, \mathrm{CH}), \\
3.54-3.61(\mathrm{~m}, 1 \mathrm{H}, 3 \alpha-\mathrm{CH}), 5.60-5.65(\mathrm{~m}, 1 \mathrm{H}, \mathrm{CH}, \mathrm{C}-6), 9.98 \\
\left(\mathrm{~s}, 1 \mathrm{H}, \mathrm{OH}, \text { exchangeable with } \mathrm{D}_{2} \mathrm{O}\right)\end{array}$ \\
\hline $2 b$ & $\begin{array}{l}461\left[\mathrm{M}^{+}\right] \\
28), 363 \\
(100)\end{array}$ & $\begin{array}{l}3444-3386 \\
(\mathrm{OH}), 2223 \\
(\mathrm{C} \equiv \mathrm{N}), 1630 \\
(\mathrm{C}=\mathrm{C})\end{array}$ & $\begin{array}{l}0.74\left(\mathrm{~s}, 3 \mathrm{H}, \mathrm{CH}_{3}, \mathrm{C}-19\right), 0.89\left(\mathrm{~s}, 3 \mathrm{H}, \mathrm{CH}_{3}, \mathrm{C}-18\right), 0.98-1.05 \\
(\mathrm{~m}, 1 \mathrm{H}, \mathrm{CH}), 1.16-1.25\left(\mathrm{~m}, 4 \mathrm{H}, 2 \mathrm{CH}_{2}\right), 1.41-1.57(\mathrm{~m}, 6 \mathrm{H}, \\
\left.3 \mathrm{CH}_{2}\right), 1.64-1.80\left(\mathrm{~m}, 4 \mathrm{H}, 2 \mathrm{CH}_{2}\right), 1.85-1.88\left(\mathrm{~m}, 4 \mathrm{H}, 2 \mathrm{CH}_{2}\right), \\
1.96-2.05(\mathrm{~m}, 1 \mathrm{H}, \mathrm{CH}), 2.34,2.36\left(2 \mathrm{~s}, 6 \mathrm{H}, 2 \times \mathrm{N}-\mathrm{CH}_{3}\right), \\
2.45-2.50\left(\mathrm{~m}, 2 \mathrm{H}, \mathrm{CH}_{2}\right), 2.56-2.60(\mathrm{~m}, 1 \mathrm{H}, \mathrm{CH}), 3.57-3.64 \\
(\mathrm{~m}, 1 \mathrm{H}, 3 \alpha-\mathrm{CH}), 5.58-5.66(\mathrm{~m}, 1 \mathrm{H}, \mathrm{CH}, \mathrm{C}-6), 7.18-7.46(\mathrm{~m}, \\
5 \mathrm{H}, \mathrm{Ar}-\mathrm{H}), 9.96\left(\mathrm{~s}, 1 \mathrm{H}, \mathrm{OH}, \text { exchangeable with } \mathrm{D}_{2} \mathrm{O}\right)\end{array}$ \\
\hline $3 a$ & $\begin{array}{l}425\left[\mathrm{M}^{+}\right] \\
(33), 260 \\
(100)\end{array}$ & $\begin{array}{l}3458-3377 \\
(\mathrm{OH}), 2226 \\
(\mathrm{C} \equiv \mathrm{N}), 1634 \\
(\mathrm{C}=\mathrm{C})\end{array}$ & $\begin{array}{l}0.75\left(\mathrm{~s}, 3 \mathrm{H}, \mathrm{CH}_{3}, \mathrm{C}-19\right), 0.91\left(\mathrm{~s}, 3 \mathrm{H}, \mathrm{CH}_{3}, \mathrm{C}-18\right), 0.96-1.00 \\
(\mathrm{~m}, 1 \mathrm{H}, \mathrm{CH}), 1.10-1.23\left(\mathrm{~m}, 4 \mathrm{H}, 2 \mathrm{CH}_{2}\right), 1.38-1.55(\mathrm{~m}, 6 \mathrm{H}, \\
\left.3 \mathrm{CH}_{2}\right), 1.58-1.62\left(\mathrm{~m}, 6 \mathrm{H}, 3 \mathrm{CH}_{2}\right), 1.65-1.79\left(\mathrm{~m}, 4 \mathrm{H}, 2 \mathrm{CH}_{2}\right), \\
1.97-2.00(\mathrm{~m}, 1 \mathrm{H}, \mathrm{CH}), 2.20-2.26\left(\mathrm{~m}, 4 \mathrm{H}, 2 \mathrm{CH}_{2}\right), 2.36(\mathrm{~s}, \\
\left.3 \mathrm{H}, \mathrm{N}-\mathrm{CH}_{3}\right), 2.44-2.48\left(\mathrm{~m}, 2 \mathrm{H}, \mathrm{CH}_{2}\right), 2.55-2.60(\mathrm{~m}, 1 \mathrm{H}, \\
\mathrm{CH}), 3.42\left(\mathrm{~s}, 2 \mathrm{H}, \mathrm{N}-\mathrm{CH}_{2}-\mathrm{N}\right), 3.54-3.61(\mathrm{~m}, 1 \mathrm{H}, 3 \alpha-\mathrm{CH}), \\
5.60-5.65(\mathrm{~m}, 1 \mathrm{H}, \mathrm{CH}, \mathrm{C}-6), 10.03(\mathrm{~s}, 1 \mathrm{H}, \mathrm{OH}, \text { exchangeab- } \\
\left.\text { le with } \mathrm{D}_{2} \mathrm{O}\right)\end{array}$ \\
\hline $3 b$ & $\begin{array}{l}487\left[\mathrm{M}^{+}\right] \\
(8), 345 \\
(100)\end{array}$ & $\begin{array}{l}3455-3396 \\
(\mathrm{OH}), 2225 \\
(\mathrm{C} \equiv \mathrm{N}), 1635 \\
(\mathrm{C}=\mathrm{C})\end{array}$ & $\begin{array}{l}0.79\left(\mathrm{~s}, 3 \mathrm{H}, \mathrm{CH}_{3}, \mathrm{C}-19\right), 0.95\left(\mathrm{~s}, 3 \mathrm{H}, \mathrm{CH}_{3}, \mathrm{C}-18\right), 0.98-1.02 \\
(\mathrm{~m}, 1 \mathrm{H}, \mathrm{CH}), 1.10-1.23\left(\mathrm{~m}, 4 \mathrm{H}, 2 \mathrm{CH}_{2}\right), 1.38-1.55(\mathrm{~m}, 6 \mathrm{H}, \\
\left.3 \mathrm{CH}_{2}\right), 1.56-1.60\left(\mathrm{~m}, 6 \mathrm{H}, 3 \mathrm{CH}_{2}\right), 1.66-1.79\left(\mathrm{~m}, 4 \mathrm{H}, 2 \mathrm{CH}_{2}\right), \\
1.95-2.05(\mathrm{~m}, 1 \mathrm{H}, \mathrm{CH}), 2.24-2.28\left(\mathrm{~m}, 4 \mathrm{H}, 2 \mathrm{CH}_{2}\right), 2.42-2.46 \\
\left(\mathrm{~m}, 2 \mathrm{H}, \mathrm{CH}_{2}\right), 2.53-2.62(\mathrm{~m}, 1 \mathrm{H}, \mathrm{CH}), 3.40(\mathrm{~s}, 2 \mathrm{H}, \\
\left.\mathrm{N}-\mathrm{CH}_{2}-\mathrm{N}\right), 3.52-3.64(\mathrm{~m}, 1 \mathrm{H}, 3 \alpha-\mathrm{CH}), 5.56-5.61(\mathrm{~m}, 1 \mathrm{H}, \\
\mathrm{CH}, \mathrm{C}-6), 7.18-7.46(\mathrm{~m}, 5 \mathrm{H}, \mathrm{Ar}-\mathrm{H}), 10.02(\mathrm{~s}, 1 \mathrm{H}, \mathrm{OH}, \mathrm{ex}- \\
\left.\text { changeable with } \mathrm{D}_{2} \mathrm{O}\right)\end{array}$ \\
\hline
\end{tabular}


4a $412\left[\mathrm{M}^{+}\right] \quad 2224(\mathrm{C} \equiv \mathrm{N}), \quad 0.77\left(\mathrm{~s}, 3 \mathrm{H}, \mathrm{CH}_{3}, \mathrm{C}-19\right), 0.92\left(\mathrm{~s}, 3 \mathrm{H}, \mathrm{CH}_{3}, \mathrm{C}-18\right), 1.00-1.05$ (32), $2601742\left(\mathrm{CO}, \quad(\mathrm{m}, 1 \mathrm{H}, \mathrm{CH}), 1.18-1.26\left(\mathrm{~m}, 4 \mathrm{H}, 2 \mathrm{CH}_{2}\right), 1.40-1.55(\mathrm{~m}, 6 \mathrm{H}\right.$, (100) ester), $\left.17283 \mathrm{CH}_{2}\right), 1.63-1.78\left(\mathrm{~m}, 4 \mathrm{H}, 2 \mathrm{CH}_{2}\right), 1.95-2.00(\mathrm{~m}, 1 \mathrm{H}, \mathrm{CH})$, $\left(\mathrm{COCH}_{3}\right), \quad 2.23\left(\mathrm{~s}, 3 \mathrm{H}, \mathrm{CO}-\mathrm{CH}_{3}\right), 2.34\left(\mathrm{~s}, 3 \mathrm{H}, \mathrm{N}-\mathrm{CH}_{3}\right), 2.40-2.50(\mathrm{~m}$, $\left.1632(\mathrm{C}=\mathrm{C}) \quad 2 \mathrm{H}, \mathrm{CH}_{2}\right), 3.65\left(\mathrm{~s}, 3 \mathrm{H}, \mathrm{COO}-\mathrm{CH}_{3}\right), 2.56-2.62(\mathrm{~m}, 1 \mathrm{H}, \mathrm{CH})$,

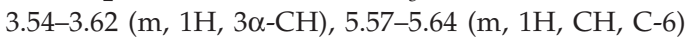
(m, 1H, CH), 1.16-1.24 (m, 4H, 2CH $), 1.43-1.53(\mathrm{~m}, 6 \mathrm{H}$, $\left.3 \mathrm{CH}_{2}\right), 1.62-1.80\left(\mathrm{~m}, 4 \mathrm{H}, 2 \mathrm{CH}_{2}\right), 1.98-2.04(\mathrm{~m}, 1 \mathrm{H}, \mathrm{CH})$, $2.25\left(\mathrm{~s}, 3 \mathrm{H}, \mathrm{CO}-\mathrm{CH}_{3}\right), 2.41-2.53\left(\mathrm{~m}, 2 \mathrm{H}, \mathrm{CH}_{2}\right), 3.66(\mathrm{~s}, 3 \mathrm{H}$, $\left.\mathrm{COO}-\mathrm{CH}_{3}\right), 2.58-2.62(\mathrm{~m}, 1 \mathrm{H}, \mathrm{CH}), 3.52-3.56(\mathrm{~m}, 1 \mathrm{H}$, $3 \alpha-\mathrm{CH}), 5.52-5.60(\mathrm{~m}, 1 \mathrm{H}, \mathrm{CH}, \mathrm{C}-6), 7.20-7.34(\mathrm{~m}, 5 \mathrm{H}$, Ar-H)

5a $520\left[\mathrm{M}^{+}\right] \quad 2226(\mathrm{C} \equiv \mathrm{N})$, (16), $2441748(\mathrm{CO}$, (100) ester), 1732 $(\mathrm{CO}$, $\left.\mathrm{COCF}_{3}\right)$ $1635(\mathrm{C}=\mathrm{C})$

0.79 (s, 3H, $\left.\mathrm{CH}_{3}, \mathrm{C}-19\right), 0.98$ (s, 3H, $\left.\mathrm{CH}_{3}, \mathrm{C}-18\right), 1.05-1.10$ $(\mathrm{m}, 1 \mathrm{H}, \mathrm{CH}), 1.20-1.27\left(\mathrm{~m}, 4 \mathrm{H}, 2 \mathrm{CH}_{2}\right), 1.45-1.54(\mathrm{~m}, 6 \mathrm{H}$, $\left.3 \mathrm{CH}_{2}\right), 1.65-1.82\left(\mathrm{~m}, 4 \mathrm{H}, 2 \mathrm{CH}_{2}\right), 1.96-2.05(\mathrm{~m}, 1 \mathrm{H}, \mathrm{CH})$, $2.35\left(\mathrm{~s}, 3 \mathrm{H}, \mathrm{N}-\mathrm{CH}_{3}\right), 2.44-2.48\left(\mathrm{~m}, 2 \mathrm{H}, \mathrm{CH}_{2}\right), 2.53-2.61(\mathrm{~m}$, $1 \mathrm{H}, \mathrm{CH}), 3.53-3.64(\mathrm{~m}, 1 \mathrm{H}, 3 \alpha-\mathrm{CH}), 5.54-5.63(\mathrm{~m}, 1 \mathrm{H}, \mathrm{CH}$ C-6) $2228(\mathrm{C} \equiv \mathrm{N})$, $1747(\mathrm{CO}$,

0.79 (s, 3H, $\left.\mathrm{CH}_{3}, \mathrm{C}-19\right), 0.92$ (s, 3H, $\left.\mathrm{CH}_{3}, \mathrm{C}-18\right), 0.98-1.06$ (100) $\left.\mathrm{COCF}_{3}\right)$, $(\mathrm{m}, 1 \mathrm{H}, \mathrm{CH}), 1.14-1.25\left(\mathrm{~m}, 4 \mathrm{H}, 2 \mathrm{CH}_{2}\right), 1.45-1.56(\mathrm{~m}, 6 \mathrm{H}$, $\left.3 \mathrm{CH}_{2}\right), 1.60-1.81\left(\mathrm{~m}, 4 \mathrm{H}, 2 \mathrm{CH}_{2}\right), 1.96-2.00(\mathrm{~m}, 1 \mathrm{H}, \mathrm{CH})$, 2.43-2.52 (m, 2H, $\left.\mathrm{CH}_{2}\right), 2.59-2.64(\mathrm{~m}, 1 \mathrm{H}, \mathrm{CH}), 3.54-3.58$ $1636(\mathrm{C}=\mathrm{C})$ $(\mathrm{m}, 1 \mathrm{H}, 3 \alpha-\mathrm{CH}), 5.52-5.59(\mathrm{~m}, 1 \mathrm{H}, \mathrm{CH}, \mathrm{C}-6), 7.22-7.35$ (m, $5 \mathrm{H}, \mathrm{Ar}-\mathrm{H})$

6a $332\left[\mathrm{M}^{+}\right] \quad 3580-3490$ (52), $244 \quad(\mathrm{OH})$, (100) 3448-3376 0.81 (s, 3H, $\left.\mathrm{CH}_{3}, \mathrm{C}-19\right), 0.96$ (s, 3H, $\left.\mathrm{CH}_{3}, \mathrm{C}-18\right), 0.99-1.08$ $(\mathrm{m}, 1 \mathrm{H}, \mathrm{CH}), 1.22-1.26\left(\mathrm{~m}, 4 \mathrm{H}, 2 \mathrm{CH}_{2}\right), 1.41-1.55(\mathrm{~m}, 6 \mathrm{H}$, $\left.3 \mathrm{CH}_{2}\right), 1.62-1.79\left(\mathrm{~m}, 4 \mathrm{H}, 2 \mathrm{CH}_{2}\right), 1.98-2.02(\mathrm{~m}, 1 \mathrm{H}, \mathrm{CH})$, $\left(\mathrm{NH}_{2}, \mathrm{NH}\right), \quad 2.36\left(\mathrm{~s}, 3 \mathrm{H}, \mathrm{N}-\mathrm{CH}_{3}\right), 2.40-2.45\left(\mathrm{~m}, 2 \mathrm{H}, \mathrm{CH}_{2}\right), 2.52-2.57(\mathrm{~m}$, $1634(\mathrm{C}=\mathrm{C}) \quad 1 \mathrm{H}, \mathrm{CH}), 3.39\left(\mathrm{~s}, 2 \mathrm{H}, \mathrm{CH}_{2}-\mathrm{N}\right), 3.50-3.61(\mathrm{~m}, 1 \mathrm{H}, 3 \alpha-\mathrm{CH})$, 3.75 (s, 2H, $\mathrm{NH}_{2}$, exchangeable with $\left.\mathrm{D}_{2} \mathrm{O}\right), 3.98(\mathrm{~s}, 1 \mathrm{H}$, $\mathrm{NH}$, exchangeable with $\left.\mathrm{D}_{2} \mathrm{O}\right), 5.58-5.62(\mathrm{~m}, 1 \mathrm{H}, \mathrm{CH}, \mathrm{C}-6)$, $9.99\left(\mathrm{~s}, 1 \mathrm{H}, \mathrm{OH}\right.$, exchangeable with $\mathrm{D}_{2} \mathrm{O}$ ) 0.79 (s, 3H, $\left.\mathrm{CH}_{3}, \mathrm{C}-19\right), 0.94$ (s, 3H, $\left.\mathrm{CH}_{3}, \mathrm{C}-18\right), 0.98-1.05$ (14), 274 $(\mathrm{OH})$, (100) $\begin{array}{ll}3442-3388 & \left.3 \mathrm{CH}_{2}\right), 1.64-1.83\left(\mathrm{~m}, 4 \mathrm{H}, 2 \mathrm{CH}_{2}\right), 1.96-2.04(\mathrm{~m}, 1 \mathrm{H}, \mathrm{CH}), \\ \left(\mathrm{NH}_{2}, \mathrm{NH}\right), & 2.41-2.47\left(\mathrm{~m}, 2 \mathrm{H}, \mathrm{CH}_{2}\right), 2.54-2.59(\mathrm{~m}, 1 \mathrm{H}, \mathrm{CH}), 3.38(\mathrm{~s},\end{array}$ $(\mathrm{m}, 1 \mathrm{H}, \mathrm{CH}), 1.24-1.28\left(\mathrm{~m}, 4 \mathrm{H}, 2 \mathrm{CH}_{2}\right), 1.42-1.58(\mathrm{~m}, 6 \mathrm{H}$, $\left.1635(\mathrm{C}=\mathrm{C}) \quad 2 \mathrm{H}, \mathrm{CH}_{2}-\mathrm{N}\right), 3.53-3.64(\mathrm{~m}, 1 \mathrm{H}, 3 \alpha-\mathrm{CH}), 3.76\left(\mathrm{~s}, 2 \mathrm{H}, \mathrm{NH}_{2}\right.$, exchangeable with $\left.\mathrm{D}_{2} \mathrm{O}\right), 3.99(\mathrm{~s}, 1 \mathrm{H}, \mathrm{NH}$, exchangeable with $\left.\mathrm{D}_{2} \mathrm{O}\right), 5.56-5.63(\mathrm{~m}, 1 \mathrm{H}, \mathrm{CH}, \mathrm{C}-6), 7.21-7.36(\mathrm{~m}, 5 \mathrm{H}$, Ar- $\mathrm{H}), 9.98$ (s, 1H, OH, exchangeable with $\left.\mathrm{D}_{2} \mathrm{O}\right)$

7a $\quad$ MS $m / z \quad 3560-3480$ 0.80 (s, 3H, $\left.\mathrm{CH}_{3}, \mathrm{C}-19\right), 0.92$ (s, 3H, $\left.\mathrm{CH}_{3}, \mathrm{C}-18\right), 0.99-1.02$ $(\%): 334 \quad(\mathrm{OH})$, $\left[\mathrm{M}^{+}\right](26), \quad 3440-3388$ (m, 1H, CH), 1.16-1.27 (m, 4H, 2CH $), 1.39-1.57(\mathrm{~m}, 6 \mathrm{H}$, $\left.3 \mathrm{CH}_{2}\right), 1.63-1.81\left(\mathrm{~m}, 6 \mathrm{H}, 3 \mathrm{CH}_{2}\right), 1.97-2.05(\mathrm{~m}, 1 \mathrm{H}, \mathrm{CH})$, $260(100) \quad\left(\mathrm{NH}_{2}, \mathrm{NH}\right)$ $2.34\left(\mathrm{~s}, 3 \mathrm{H}, \mathrm{N}-\mathrm{CH}_{3}\right), 2.44-2.48\left(\mathrm{~m}, 2 \mathrm{H}, \mathrm{CH}_{2}\right), 2.56-2.65(\mathrm{~m}$, $1 \mathrm{H}, \mathrm{CH}), 3.00-3.10(\mathrm{~m}, 1 \mathrm{H}, 5 \delta-\mathrm{CH}), 3.37\left(\mathrm{~s}, 2 \mathrm{H}, \mathrm{CH}_{2}-\mathrm{N}\right)$, 3.53-3.60 (m, $1 \mathrm{H}, 3 \alpha-\mathrm{CH}), 3.80\left(\mathrm{~s}, 2 \mathrm{H}, \mathrm{NH}_{2}\right.$, exchangeable with $\left.\mathrm{D}_{2} \mathrm{O}\right), 4.02\left(\mathrm{~s}, 1 \mathrm{H}, \mathrm{NH}\right.$, exchangeable with $\left.\mathrm{D}_{2} \mathrm{O}\right), 9.98$ $\left(\mathrm{s}, 1 \mathrm{H}, \mathrm{OH}\right.$, exchangeable with $\mathrm{D}_{2} \mathrm{O}$ ) 

58 (2008) $43-59$.

\begin{tabular}{|c|c|c|c|}
\hline $7 b$ & $\begin{array}{l}396\left[\mathrm{M}^{+}\right] \\
(33), 256 \\
(100)\end{array}$ & $\begin{array}{l}3566-3477 \\
(\mathrm{OH}), \\
3443-3386 \\
\left(\mathrm{NH}_{2}, \mathrm{NH}\right)\end{array}$ & $\begin{array}{l}0.79\left(\mathrm{~s}, 3 \mathrm{H}, \mathrm{CH}_{3}, \mathrm{C}-19\right), 0.88\left(\mathrm{~s}, 3 \mathrm{H}, \mathrm{CH}_{3}, \mathrm{C}-18\right), 0.97-1.00 \\
(\mathrm{~m}, 1 \mathrm{H}, \mathrm{CH}), 1.15-1.25\left(\mathrm{~m}, 4 \mathrm{H}, 2 \mathrm{CH}_{2}\right), 1.42-1.56(\mathrm{~m}, 6 \mathrm{H}, \\
\left.3 \mathrm{CH}_{2}\right), 1.61-1.80\left(\mathrm{~m}, 6 \mathrm{H}, 3 \mathrm{CH}_{2}\right), 1.99-2.06(\mathrm{~m}, 1 \mathrm{H}, \mathrm{CH}), \\
2.43-2.49\left(\mathrm{~m}, 2 \mathrm{H}, \mathrm{CH}_{2}\right), 2.57-2.64(\mathrm{~m}, 1 \mathrm{H}, \mathrm{CH}), 3.05-3.15 \\
(\mathrm{~m}, 1 \mathrm{H}, 5 \delta-\mathrm{CH}), 3.35\left(\mathrm{~s}, 2 \mathrm{H}, \mathrm{CH}_{2}-\mathrm{N}\right), 3.54-3.62(\mathrm{~m}, 1 \mathrm{H}, \\
3 \alpha-\mathrm{CH}), 3.78\left(\mathrm{~s}, 2 \mathrm{H}, \mathrm{NH}_{2} \text {, exchangeable with } \mathrm{D}_{2} \mathrm{O}\right), 4.00 \\
\left(\mathrm{~s}, 1 \mathrm{H}, \mathrm{NH}, \text { exchangeable with } \mathrm{D}_{2} \mathrm{O}\right), 7.22-7.35(\mathrm{~m}, 5 \mathrm{H}, \\
\text { Ar-H), } 10.00\left(\mathrm{~s}, 1 \mathrm{H}, \mathrm{OH} \text {, exchangeable with } \mathrm{D}_{2} \mathrm{O}\right)\end{array}$ \\
\hline $8 a$ & $\begin{array}{l}346\left[\mathrm{M}^{+}\right] \\
(10), 244 \\
(100)\end{array}$ & $\begin{array}{l}3560-3474 \\
(\mathrm{OH}) \\
3442-3388 \\
\left(\mathrm{NH}_{2}, \mathrm{NH}\right) \\
1660(\mathrm{C}=\mathrm{O}, \\
\text { amide }), 1636 \\
(\mathrm{C}=\mathrm{C})\end{array}$ & $\begin{array}{l}0.79\left(\mathrm{~s}, 3 \mathrm{H}, \mathrm{CH}_{3}, \mathrm{C}-19\right), 0.94\left(\mathrm{~s}, 3 \mathrm{H}, \mathrm{CH}_{3}, \mathrm{C}-18\right), 0.95-1.05 \\
(\mathrm{~m}, 1 \mathrm{H}, \mathrm{CH}), 1.16-1.25\left(\mathrm{~m}, 4 \mathrm{H}, 2 \mathrm{CH}_{2}\right), 1.44-1.54(\mathrm{~m}, 6 \mathrm{H}, \\
\left.3 \mathrm{CH}_{2}\right), 1.61-1.82\left(\mathrm{~m}, 4 \mathrm{H}, 2 \mathrm{CH}_{2}\right), 1.96-2.05(\mathrm{~m}, 1 \mathrm{H}, \mathrm{CH}), \\
2.33\left(\mathrm{~s}, 3 \mathrm{H}, \mathrm{N}-\mathrm{CH}_{3}\right), 2.42-2.49\left(\mathrm{~m}, 2 \mathrm{H}, \mathrm{CH}_{2}\right), 2.57-2.64(\mathrm{~m}, \\
1 \mathrm{H}, \mathrm{CH}), 3.52-3.63(\mathrm{~m}, 1 \mathrm{H}, 3 \alpha-\mathrm{CH}), 3.74\left(\mathrm{~s}, 2 \mathrm{H}, \mathrm{NH}_{2} \text {, ex- }\right. \\
\left.\text { changeable with } \mathrm{D}_{2} \mathrm{O}\right), 3.98(\mathrm{~s}, 1 \mathrm{H}, \mathrm{NH}, \text { exchangeable wi- } \\
\left.\text { th } \mathrm{D}_{2} \mathrm{O}\right), 5.56-5.61(\mathrm{~m}, 1 \mathrm{H}, \mathrm{CH}, \mathrm{C}-6), 9.86(\mathrm{~s}, 1 \mathrm{H}, \mathrm{OH}, \text { ex- } \\
\left.\text { changeable with } \mathrm{D}_{2} \mathrm{O}\right)\end{array}$ \\
\hline $8 b$ & $\begin{array}{l}408\left[\mathrm{M}^{+}\right] \\
(18), 256 \\
(100)\end{array}$ & $\begin{array}{l}3569-3480 \\
(\mathrm{OH}) \\
3439-3392 \\
\left(\mathrm{NH}_{2}, \mathrm{NH}\right) \\
1662(\mathrm{C}=\mathrm{O}, \\
\text { amide }), 1635 \\
(\mathrm{C}=\mathrm{C})\end{array}$ & $\begin{array}{l}0.81\left(\mathrm{~s}, 3 \mathrm{H}, \mathrm{CH}_{3}, \mathrm{C}-19\right), 0.93\left(\mathrm{~s}, 3 \mathrm{H}, \mathrm{CH}_{3}, \mathrm{C}-18\right), 0.99-1.05 \\
(\mathrm{~m}, 1 \mathrm{H}, \mathrm{CH}), 1.15-1.24\left(\mathrm{~m}, 4 \mathrm{H}, 2 \mathrm{CH}_{2}\right), 1.43-1.57(\mathrm{~m}, 6 \mathrm{H}, \\
\left.3 \mathrm{CH}_{2}\right), 1.65-1.80\left(\mathrm{~m}, 4 \mathrm{H}, 2 \mathrm{CH}_{2}\right), 1.98-2.02(\mathrm{~m}, 1 \mathrm{H}, \mathrm{CH}), \\
2.40-2.48\left(\mathrm{~m}, 2 \mathrm{H}, \mathrm{CH}_{2}\right), 2.54-2.61(\mathrm{~m}, 1 \mathrm{H}, \mathrm{CH}), 3.50-3.64 \\
(\mathrm{~m}, 1 \mathrm{H}, 3 \alpha-\mathrm{CH}), 3.86\left(\mathrm{~s}, 2 \mathrm{H}, \mathrm{NH}_{2} \text {, exchangeable with }\right. \\
\left.\mathrm{D}_{2} \mathrm{O}\right), 3.97\left(\mathrm{~s}, 1 \mathrm{H}, \mathrm{NH}, \text { exchangeable with } \mathrm{D}_{2} \mathrm{O}\right), 5.53-5.60 \\
(\mathrm{~m}, 1 \mathrm{H}, \mathrm{CH}, \mathrm{C}-6), 7.18-7.38(\mathrm{~m}, 5 \mathrm{H}, \mathrm{Ar}-\mathrm{H}), 9.98(\mathrm{~s}, 1 \mathrm{H}, \\
\left.\text { OH, exchangeable with } \mathrm{D}_{2} \mathrm{O}\right)\end{array}$ \\
\hline $9 a$ & $\begin{array}{l}347\left[\mathrm{M}^{+}\right] \\
(14), 301 \\
(100)\end{array}$ & $\begin{array}{l}3472-3396 \\
(\mathrm{OH}, \mathrm{NH}) \\
1718(\mathrm{CO}, \\
\text { acid), } 1634 \\
(\mathrm{C}=\mathrm{C})\end{array}$ & $\begin{array}{l}0.78\left(\mathrm{~s}, 3 \mathrm{H}, \mathrm{CH}_{3}, \mathrm{C}-19\right), 0.92\left(\mathrm{~s}, 3 \mathrm{H}, \mathrm{CH}_{3}, \mathrm{C}-18\right), 0.97-1.06 \\
(\mathrm{~m}, 1 \mathrm{H}, \mathrm{CH}), 1.18-1.26\left(\mathrm{~m}, 4 \mathrm{H}, 2 \mathrm{CH}_{2}\right), 1.43-1.57(\mathrm{~m}, 6 \mathrm{H}, \\
\left.3 \mathrm{CH}_{2}\right), 1.60-1.80\left(\mathrm{~m}, 4 \mathrm{H}, 2 \mathrm{CH}_{2}\right), 1.95-2.00(\mathrm{~m}, 1 \mathrm{H}, \mathrm{CH}), \\
2.32\left(\mathrm{~s}, 3 \mathrm{H}, \mathrm{N}-\mathrm{CH}_{3}\right), 2.40-2.47\left(\mathrm{~m}, 2 \mathrm{H}, \mathrm{CH}_{2}\right), 2.55-2.62(\mathrm{~m}, \\
1 \mathrm{H}, \mathrm{CH}), 3.53-3.62(\mathrm{~m}, 1 \mathrm{H}, 3 \alpha-\mathrm{CH}), 4.02(\mathrm{~s}, 1 \mathrm{H}, \mathrm{NH}, \mathrm{ex}- \\
\left.\text { changeable with } \mathrm{D}_{2} \mathrm{O}\right), 5.55-5.60(\mathrm{~m}, 1 \mathrm{H}, \mathrm{CH}, \mathrm{C}-6), \\
\left.9.86-10.56 \text { (br. s, } 2 \mathrm{H}, 2 \times \mathrm{OH}, \text { exchangeable with } \mathrm{D}_{2} \mathrm{O}\right)\end{array}$ \\
\hline $9 b$ & $\begin{array}{l}409\left[\mathrm{M}^{+}\right] \\
(8), 345 \\
(100)\end{array}$ & $\begin{array}{l}3444-3387 \\
(\mathrm{OH}, \mathrm{NH}), \\
1723(\mathrm{CO}, \\
\text { acid), } 1630 \\
(\mathrm{C}=\mathrm{C})\end{array}$ & $\begin{array}{l}0.81\left(\mathrm{~s}, 3 \mathrm{H}, \mathrm{CH}_{3}, \mathrm{C}-19\right), 0.97\left(\mathrm{~s}, 3 \mathrm{H}, \mathrm{CH}_{3}, \mathrm{C}-18\right), 0.99-1.04 \\
(\mathrm{~m}, 1 \mathrm{H}, \mathrm{CH}), 1.20-1.25\left(\mathrm{~m}, 4 \mathrm{H}, 2 \mathrm{CH}_{2}\right), 1.42-1.56(\mathrm{~m}, 6 \mathrm{H}, \\
\left.3 \mathrm{CH}_{2}\right), 1.61-1.79\left(\mathrm{~m}, 4 \mathrm{H}, 2 \mathrm{CH}_{2}\right), 1.96-2.02(\mathrm{~m}, 1 \mathrm{H}, \mathrm{CH}), \\
2.39-2.46\left(\mathrm{~m}, 2 \mathrm{H}, \mathrm{CH}_{2}\right), 2.53-2.60(\mathrm{~m}, 1 \mathrm{H}, \mathrm{CH}), 3.54-3.60 \\
(\mathrm{~m}, 1 \mathrm{H}, 3 \alpha-\mathrm{CH}), 4.15(\mathrm{~s}, 1 \mathrm{H}, \mathrm{NH}, \text { exchangeable with } \\
\left.\mathrm{D}_{2} \mathrm{O}\right), 5.52-5.58(\mathrm{~m}, 1 \mathrm{H}, \mathrm{CH}, \mathrm{C}-6), 7.19-7.36(\mathrm{~m}, 5 \mathrm{H}, \\
\mathrm{Ar}-\mathrm{H}), 9.88-10.60 \text { (br. s, } 2 \mathrm{H}, 2 \times \mathrm{OH} \text {, exchangeable with } \\
\left.\mathrm{D}_{2} \mathrm{O}\right)\end{array}$ \\
\hline $10 a$ & $\begin{array}{l}345\left[\mathrm{M}^{+}\right] \\
(100)\end{array}$ & $\begin{array}{l}3505-3410 \\
(\mathrm{OH}, \mathrm{NH}), \\
1735(\mathrm{CO}, \\
\text { enone), } 1719 \\
(\mathrm{CO}, \text { acid), } \\
1636(\mathrm{C}=\mathrm{C})\end{array}$ & $\begin{array}{l}0.80\left(\mathrm{~s}, 3 \mathrm{H}, \mathrm{CH}_{3}, \mathrm{C}-19\right), 0.94\left(\mathrm{~s}, 3 \mathrm{H}, \mathrm{CH}_{3}, \mathrm{C}-18\right), 0.99-1.06 \\
(\mathrm{~m}, 1 \mathrm{H}, \mathrm{CH}), 1.21-1.27\left(\mathrm{~m}, 4 \mathrm{H}, 2 \mathrm{CH}_{2}\right), 1.41-1.59(\mathrm{~m}, 6 \mathrm{H}, \\
\left.3 \mathrm{CH}_{2}\right), 1.62-1.81\left(\mathrm{~m}, 4 \mathrm{H}, 2 \mathrm{CH}_{2}\right), 1.98-2.04(\mathrm{~m}, 1 \mathrm{H}, \mathrm{CH}), \\
2.35\left(\mathrm{~s}, 3 \mathrm{H}, \mathrm{N}-\mathrm{CH}_{3}\right), 2.42-2.49\left(\mathrm{~m}, 2 \mathrm{H}, \mathrm{CH}_{2}\right), 2.54-2.60(\mathrm{~m}, \\
1 \mathrm{H}, \mathrm{CH}), 4.00\left(\mathrm{~s}, 1 \mathrm{H}, \mathrm{NH}, \text { exchangeable with } \mathrm{D}_{2} \mathrm{O}\right), \\
\text { 5.52-5.61 (m, } 1 \mathrm{H}, \mathrm{CH}, \mathrm{C}-6), 10.86 \text { (br. s, } 1 \mathrm{H}, \mathrm{OH}, \text { exchan- } \\
\left.\text { geable with } \mathrm{D}_{2} \mathrm{O}\right)\end{array}$ \\
\hline
\end{tabular}



58 (2008) 43-59.

\begin{tabular}{|c|c|c|}
\hline $\begin{array}{l}407\left[\mathrm{M}^{+}\right] \\
(15), 407 \\
(100)\end{array}$ & $\begin{array}{l}3448-3386 \\
(\mathrm{OH}, \mathrm{NH}), \\
1734(\mathrm{CO}, \\
\text { enone), } 1721 \\
(\mathrm{CO}, \text { acid), } \\
1635(\mathrm{C}=\mathrm{C})\end{array}$ & $\begin{array}{l}0.83\left(\mathrm{~s}, 3 \mathrm{H}, \mathrm{CH}_{3}, \mathrm{C}-19\right), 0.95\left(\mathrm{~s}, 3 \mathrm{H}, \mathrm{CH}_{3}, \mathrm{C}-18\right), 1.00-1.05 \\
(\mathrm{~m}, 1 \mathrm{H}, \mathrm{CH}), 1.21-1.27\left(\mathrm{~m}, 4 \mathrm{H}, 2 \mathrm{CH}_{2}\right), 1.39-1.58(\mathrm{~m}, 6 \mathrm{H}, \\
\left.3 \mathrm{CH}_{2}\right), 1.63-1.82\left(\mathrm{~m}, 4 \mathrm{H}, 2 \mathrm{CH}_{2}\right), 1.96-2.05(\mathrm{~m}, 1 \mathrm{H}, \mathrm{CH}), \\
2.40-2.46\left(\mathrm{~m}, 2 \mathrm{H}, \mathrm{CH}_{2}\right), 2.51-2.59(\mathrm{~m}, 1 \mathrm{H}, \mathrm{CH}), 3.98(\mathrm{~s}, \\
\left.1 \mathrm{H}, \mathrm{NH}, \text { exchangeable with } \mathrm{D}_{2} \mathrm{O}\right), 5.50-5.60(\mathrm{~m}, 1 \mathrm{H}, \mathrm{CH}, \\
\mathrm{C}-6), 7.21-7.35(\mathrm{~m}, 5 \mathrm{H}, \mathrm{Ar}-\mathrm{H}), 10.68 \text { (br. s, } 1 \mathrm{H}, \mathrm{OH}, \text { ex- } \\
\left.\text { changeable with } \mathrm{D}_{2} \mathrm{O}\right)\end{array}$ \\
\hline $\begin{array}{l}371\left[\mathrm{M}^{+}\right] \\
(16), 299 \\
(100)\end{array}$ & $\begin{array}{l}3454-3378 \\
(\mathrm{OH}, \mathrm{NH}), \\
1633(\mathrm{C}=\mathrm{C}), \\
1610(\mathrm{C}=\mathrm{N})\end{array}$ & $\begin{array}{l}0.77\left(\mathrm{~s}, 3 \mathrm{H}, \mathrm{CH}_{3}, \mathrm{C}-19\right), 0.94\left(\mathrm{~s}, 3 \mathrm{H}, \mathrm{CH}_{3}, \mathrm{C}-18\right), 0.99-1.06 \\
(\mathrm{~m}, 1 \mathrm{H}, \mathrm{CH}), 1.19-1.25\left(\mathrm{~m}, 4 \mathrm{H}, 2 \mathrm{CH}_{2}\right), 1.40-1.56(\mathrm{~m}, 6 \mathrm{H}, \\
\left.3 \mathrm{CH}_{2}\right), 1.61-1.83\left(\mathrm{~m}, 4 \mathrm{H}, 2 \mathrm{CH}_{2}\right), 1.97-2.00(\mathrm{~m}, 1 \mathrm{H}, \mathrm{CH}), \\
2.38\left(\mathrm{~s}, 3 \mathrm{H}, \mathrm{N}-\mathrm{CH}_{3}\right), 2.41-2.48\left(\mathrm{~m}, 2 \mathrm{H}, \mathrm{CH}_{2}\right), 2.54-2.58(\mathrm{~m}, \\
1 \mathrm{H}, \mathrm{CH}), 3.52-3.60(\mathrm{~m}, 1 \mathrm{H}, 3 \alpha-\mathrm{CH}), 3.64-3.68(\mathrm{~m}, 4 \mathrm{H}, \\
\left.2 \mathrm{CH}_{2}\right), 3.98\left(\mathrm{~s}, 1 \mathrm{H}, \mathrm{NH} \text {, exchangeable with } \mathrm{D}_{2} \mathrm{O}\right), \\
\text { 5.55-5.65 (m, 1H, CH, C-6), 8.40 (s, 1H, NH, exchangeable } \\
\left.\text { with } \mathrm{D}_{2} \mathrm{O}\right), 10.06\left(\mathrm{~s}, 1 \mathrm{H}, \mathrm{OH} \text {, exchangeable with } \mathrm{D}_{2} \mathrm{O}\right)\end{array}$ \\
\hline $\begin{array}{l}433\left[\mathrm{M}^{+}\right] \\
(26), 274 \\
(100)\end{array}$ & $\begin{array}{l}3462-3386 \\
(\mathrm{OH}, \mathrm{NH}) \\
1636(\mathrm{C}=\mathrm{C}) \\
1605(\mathrm{C}=\mathrm{N})\end{array}$ & $\begin{array}{l}0.79\left(\mathrm{~s}, 3 \mathrm{H}, \mathrm{CH}_{3}, \mathrm{C}-19\right), 0.87\left(\mathrm{~s}, 3 \mathrm{H}, \mathrm{CH}_{3}, \mathrm{C}-18\right), 0.97-1.04 \\
(\mathrm{~m}, 1 \mathrm{H}, \mathrm{CH}), 1.16-1.25\left(\mathrm{~m}, 4 \mathrm{H}, 2 \mathrm{CH}_{2}\right), 1.40-1.54(\mathrm{~m}, 6 \mathrm{H}, \\
\left.3 \mathrm{CH}_{2}\right), 1.60-1.80\left(\mathrm{~m}, 4 \mathrm{H}, 2 \mathrm{CH}_{2}\right), 1.98-2.03(\mathrm{~m}, 1 \mathrm{H}, \mathrm{CH}), \\
2.42-2.50\left(\mathrm{~m}, 2 \mathrm{H}, \mathrm{CH}_{2}\right), 2.58-2.64(\mathrm{~m}, 1 \mathrm{H}, \mathrm{CH}), 3.55-3.61 \\
(\mathrm{~m}, 1 \mathrm{H}, 3 \alpha-\mathrm{CH}), 3.66-3.69\left(\mathrm{~m}, 4 \mathrm{H}, 2 \mathrm{CH}_{2}\right), 4.00(\mathrm{~s}, 1 \mathrm{H}, \mathrm{NH}, \\
\left.\text { exchangeable with } \mathrm{D}_{2} \mathrm{O}\right), 5.51-5.62(\mathrm{~m}, 1 \mathrm{H}, \mathrm{CH}, \mathrm{C}-6), \\
\text { 7.19-7.38 (m, 5H, Ar-H), } 8.32(\mathrm{~s}, 1 \mathrm{H}, \mathrm{NH}, \text { exchangeable } \\
\left.\text { with } \mathrm{D}_{2} \mathrm{O}\right), 10.00\left(\mathrm{~s}, 1 \mathrm{H}, \mathrm{OH} \text {, exchangeable with } \mathrm{D}_{2} \mathrm{O}\right)\end{array}$ \\
\hline
\end{tabular}

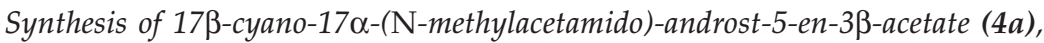

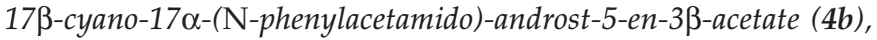

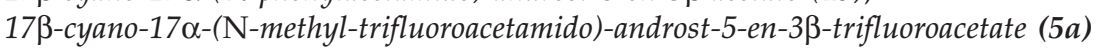

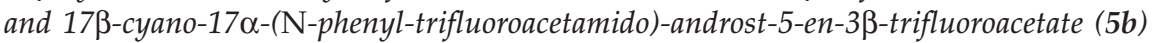

A solution of compounds $\mathbf{1 a}, \mathbf{b}(150 \mathrm{mg})$ in acetyl chloride or trifluoroacetic anhydride $(5 \mathrm{~mL})$ was stirred at room temperature for 24 hours. The reaction mixture was poured into ice-water, the separated solid was filtered off, dried and crystallized from the proper solvent to yield the compounds $\mathbf{4} \mathbf{a}, \mathbf{b}$ and $\mathbf{5 a}, \mathbf{b}$, respectively (Scheme 2 ).

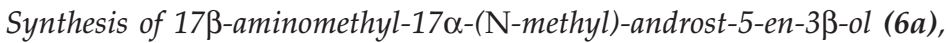

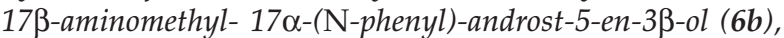

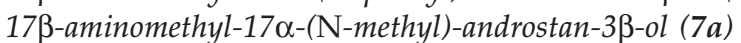

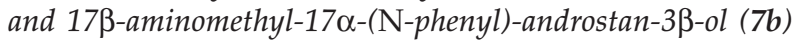

A mixture of compounds $\mathbf{1} \mathbf{a}, \mathbf{b}(5 \mathrm{mmol})$ and catalytic reagents, namely, palladium-charcoal or platinum oxide-charcoal (Adam's catalyst) $(50 \mathrm{mg})$ in absolute ethanol (25 $\mathrm{mL}$ ) was shaken at $75^{\circ} \mathrm{C}$ under hydrogen $\left(5 \times 10^{5} \mathrm{~Pa}\right)$ for 7 hours and filtered. The filtrate was evaporated under reduced pressure, the residue was solidified with ether, the separated solid was filtered off, dried and crystallized from the proper solvent to yield the compounds $\mathbf{6} \mathbf{a}, \mathbf{b}$ and $\mathbf{7 a}, \mathbf{b}$, respectively (Scheme 2 ). 


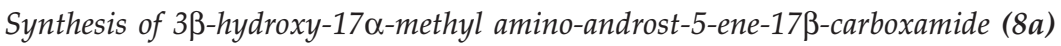

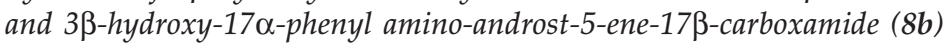

A solution of compounds $1 \mathbf{a}, \mathbf{b}(1 \mathrm{mmol})$ in sulphuric acid $(0.5 \mathrm{~mol} \mathrm{~L}-1,10 \mathrm{~mL})$ was heated at $50{ }^{\circ} \mathrm{C}$ for 5 hours, then left overnight at room temperature. The reaction mixture was poured into ice water, the separated product was filtered off, washed with water, dried and crystallized from the proper solvent to give the corresponding carboxamide derivatives $\mathbf{8 a}, \mathbf{b}$, respectively (Scheme 3 ).

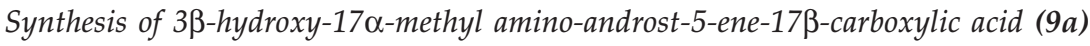

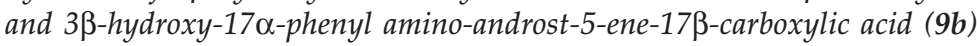

A mixture of compounds $\mathbf{1 a}, \mathbf{b}$ (1 mmol) in alcoholic sodium hydroxide $(25 \mathrm{~mL}, 10 \%)$ was refluxed for 7 hours. The reaction mixture was acidified with $1 \mathrm{~mol} \mathrm{~L}^{-1}$ hydrochloric acid $(\mathrm{pH} \sim 3)$. The separated product was filtered off, washed with water, dried and crystallized from the proper solvent to give the corresponding acid derivatives $\mathbf{9 a}, \mathbf{b}$, respectively (Scheme 3).

Synthesis of 3-oxo-17 $\alpha$-methyl amino-androst-5-ene-17 $\beta$-carboxylic acid (10a)

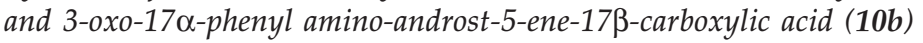

A mixture of compounds $\mathbf{1 a}, \mathbf{b}(2 \mathrm{mmol})$ and Killian reagent $(6 \mathrm{~mL})$ [prepared from potassium dichromate $(6 \mathrm{~g})$ and concentrated sulfuric acid $(8 \mathrm{~mL})$ in water $(27 \mathrm{~mL})]$ in glacial acetic acid $(15 \mathrm{~mL})$ was stirred at room temperature for 1.5 hours. The reaction mixture was warmed with methanol $(15 \mathrm{~mL})$ to destroy excess chromic acid, then it was poured into ice-water; the solid formed was filtered off, washed with water, dried and crystallized from the proper solvent to give 3-oxo-17-substituted amino-androst-5-ene17-carboxylic acids 10a,b, respectively (Scheme 3).

Synthesis of $17 \alpha-(2$-imidazolyl)-17 $\beta$-methyl amino-androst-5-ene-3 $\beta$-ol (11a)

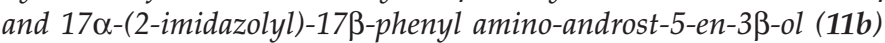

A mixture of compounds $\mathbf{1} \mathbf{a}, \mathbf{b}(1 \mathrm{mmol})$ in ethylene diamine $(15 \mathrm{~mL})$ was refluxed for 3 hours. The reaction mixture was poured into water, the formed solid was filtered off and crystallized from the proper solvent to give the corresponding imidazole derivatives $\mathbf{1 1} \mathbf{a}, \mathbf{b}$, respectively (Scheme 3 ).

\section{Biological screening}

Animals. - Biological experiments were made according to the ethical rules and animals were obtained from the animal house colony of the National Research Center, Cairo, Egypt. All animals were allowed free access to water and were kept on a constant standard diet. Prepubertal Sprague-Dawley male albino rats 21 day old (45-50 g), were used to investigate the effect of the tested compounds on the development of male sex organs. Adult male albino rats (150 days) weighting (150-200 g) were used in the present work to evaluate the androgenic-anabolic activity of all newly synthesized derivatives. 

58 (2008) $43-59$.

\section{Acute toxicity $\left(\mathrm{LD}_{50}\right)$}

Male rats were used to determine intraperitoneal $L D_{50}$ of the tested compounds. Prior to determination of the $L D_{50}$ value, a range finding screen was conducted using 20 rats each treat with a tested compound at a dose ranging from 3-2000 $\mathrm{mg} \mathrm{kg}^{-1}$. Based on the mortality observed within 14 days, the doses used for the $L D_{50}$ determination were $3,10,30,100,300,1000,2000 \mathrm{mg} \mathrm{kg}^{-1}$ for compounds administered by intraperitoneal injection as a $10 \%$ solution in dimethyl sulphoxide (DMSO). Control animals received intraperitoneal injections of DMSO. For each concentration and control, ten male rats were injected with the tested compounds twice daily for two weeks to kill any swirved animals. From the mortality data of all tested animals, the intraperitoneal $L D_{50}$ values for each agent were determined according to Austen et al. (22) (Table III).

Table III. Evaluation of $\mathrm{LD}_{50}$ of the synthesized compounds

\begin{tabular}{cc}
\hline Compound & $L D_{50}\left(\mathrm{mg} \mathrm{kg}^{-1}\right)^{\mathrm{a}}$ \\
\hline Testosterone & $2751 \pm 5$ \\
Dehydroepiandrosterone & $3411 \pm 4$ \\
$\mathbf{1 a}$ & $3211 \pm 8$ \\
$\mathbf{1 b}$ & $2561 \pm 5$ \\
$\mathbf{2 a}$ & $2818 \pm 5$ \\
$\mathbf{2 b}$ & $2913 \pm 6$ \\
$\mathbf{3 a}$ & $2781 \pm 4$ \\
$\mathbf{3 b}$ & $2750 \pm 6$ \\
$\mathbf{4 a}$ & $2713 \pm 6$ \\
$\mathbf{4 b}$ & $2514 \pm 6$ \\
$\mathbf{5 a}$ & $3164 \pm 8$ \\
$\mathbf{5 b}$ & $3000 \pm 7$ \\
$\mathbf{6 a}$ & $2634 \pm 8$ \\
$\mathbf{6 b}$ & $2894 \pm 7$ \\
$\mathbf{7 a}$ & $2856 \pm 7$ \\
$\mathbf{7 b}$ & $2646 \pm 7$ \\
$\mathbf{8 a}$ & $2895 \pm 7$ \\
$\mathbf{8 b}$ & $2648 \pm 7$ \\
$\mathbf{9 a}$ & $2865 \pm 6$ \\
$\mathbf{9 b}$ & $2743 \pm 5$ \\
$\mathbf{1 0 a}$ & $2891 \pm 5$ \\
$\mathbf{1 0 b}$ & $2914 \pm 4$ \\
$\mathbf{1 1 a}$ & $2988 \pm 5$ \\
$\mathbf{1 1 b}$ & $2755 \pm 3$ \\
&
\end{tabular}

\footnotetext{
${ }^{\text {a }}$ Mean $\pm \mathrm{SD}, n=24$
} 
N. A. Abd El-Latif et al.: New steroidal derivatives synthesized using 33-hydroxyandrosten-17-one as starting material, Acta Pharm. 58 (2008) $43-59$.

\section{Androgenic-anabolic activity}

Groups of immature male albino rats (each group contains 8 animals), 21 days old, received subcutaneously particular target compounds and testosterone as reference standard at a total dose of $0.7 \mathrm{mg} \mathrm{kg}^{-1}$ according to the following design: group 1 received the vehicle (DMSO); group 2 received the testosterone reference standard in vehicle (DMSO) (at a dose of $0.1 \mathrm{mg} \mathrm{kg}^{-1}$ daily for 7 days); group 3 was subdivided into 22 subgroups, each received individually one of the tested compounds (at a dose of $0.1 \mathrm{mg}$ $\mathrm{kg}^{-1}$ daily for 7 days).

Androgenic-anabolic activity of all newly synthesized compounds was measured according to the reported procedure $(23,24)$ (Tables IV and V).

Table IV. Androgenic-anabolic activity of newly synthesized compounds

\begin{tabular}{cccc}
\hline Compound & Mass of prostate gland $(\mathrm{g})^{\mathrm{a}}$ & ${\text { Mass of levator ani-muscle }(\mathrm{g})^{\mathrm{a}}}$ & Ratio $^{\mathrm{b}}$ \\
\hline Control & $0.20 \pm 0.02$ & $0.18 \pm 0.02$ & - \\
Testosterone & $0.76 \pm 0.01$ & $0.20 \pm 0.01$ & 0.05 \\
$\mathbf{1 a}$ & $0.32 \pm 0.01$ & $0.44 \pm 0.15$ & 2.20 \\
$\mathbf{1 b}$ & $0.39 \pm 0.01$ & $0.54 \pm 0.01$ & 1.90 \\
$\mathbf{2 a}$ & $0.41 \pm 0.01$ & $0.56 \pm 0.01$ & 1.82 \\
$\mathbf{2 b}$ & $0.39 \pm 0.01$ & $0.42 \pm 0.13$ & 1.28 \\
$\mathbf{3 a}$ & $0.71 \pm 0.01$ & $0.73 \pm 0.02$ & 0.97 \\
$\mathbf{3 b}$ & $0.66 \pm 0.02$ & $0.68 \pm 0.02$ & 1.09 \\
$\mathbf{4 a}$ & $0.73 \pm 0.01$ & $0.75 \pm 0.02$ & 1.08 \\
$\mathbf{4 b}$ & $0.44 \pm 0.02$ & $0.58 \pm 0.01$ & 1.68 \\
$\mathbf{5 a}$ & $0.31 \pm 0.01$ & $0.43 \pm 0.16$ & 2.30 \\
$\mathbf{5 b}$ & $0.75 \pm 0.01$ & $0.76 \pm 0.02$ & 1.06 \\
$\mathbf{6 a}$ & $0.81 \pm 0.02$ & $0.19 \pm 0.01$ & 0.02 \\
$\mathbf{6 b}$ & $0.83 \pm 0.026$ & $0.21 \pm 0.02$ & 0.06 \\
$\mathbf{7 a}$ & $0.81 \pm 0.01$ & $0.23 \pm 0.01$ & 0.09 \\
$\mathbf{7 b}$ & $0.82 \pm 0.02$ & $0.22 \pm 0.02$ & 0.06 \\
$\mathbf{8 a}$ & $0.82 \pm 0.01$ & $0.21 \pm 0.02$ & 0.06 \\
$\mathbf{8 b}$ & $0.87 \pm 0.01$ & $0.25 \pm 0.01$ & 0.10 \\
$\mathbf{9 a}$ & $0.83 \pm 0.02$ & $0.27 \pm 0.01$ & 0.14 \\
$\mathbf{9 b}$ & $0.86 \pm 0.02$ & $0.28 \pm 0.01$ & 0.16 \\
$\mathbf{1 0 a}$ & $0.91 \pm 0.03$ & $0.30 \pm 0.03$ & 0.17 \\
$\mathbf{1 0 b}$ & $0.96 \pm 0.02$ & $0.31 \pm 0.02$ & 0.17 \\
$\mathbf{1 1 a}$ & $0.97 \pm 0.02$ & $0.30 \pm 0.02$ & 0.16 \\
$\mathbf{1 1 b}$ & $0.96 \pm 0.02$ & $0.24 \pm 0.02$ & 0.08 \\
\hline
\end{tabular}

\footnotetext{
a Mean $\pm \operatorname{SEM}(n=8)$.
}

$\mathrm{b}$ Ratio of the mass gained by the levator ani-muscle to the mass gained by the prostate gland. 
N. A. Abd El-Latif et al.: New steroidal derivatives synthesized using 3ß-hydroxyandrosten-17-one as starting material, Acta Pharm. 58 (2008) $43-59$.

Table V. Effect of synthesized compounds on androgenic organs

\begin{tabular}{ccccc}
\hline \multirow{2}{*}{ Compound } & \multicolumn{3}{c}{ Mass $(\mathrm{g})^{\mathrm{a}}$} \\
\cline { 2 - 5 } & Epididymis & Testis & Seminal vesicles & Vasdifference $^{\mathrm{c}}$ \\
\hline Testosterone & $0.36 \pm 0.03^{\mathrm{b}}$ & $0.70 \pm 0.02^{\mathrm{b}}$ & $0.63 \pm 0.01^{\mathrm{b}}$ & $0.19 \pm 0.012^{\mathrm{b}}$ \\
$\mathbf{1 a}$ & $0.31 \pm 0.04^{\mathrm{c}}$ & $0.45 \pm 0.04^{\mathrm{c}}$ & $0.59 \pm 0.01^{\mathrm{bc}}$ & $0.72 \pm 0.08^{\mathrm{c}}$ \\
$\mathbf{1 b}$ & $0.36 \pm 0.03^{\mathrm{b}}$ & $0.68 \pm 0.02$ & $0.58 \pm 0.01^{\mathrm{b}}$ & $0.19 \pm 0.01^{\mathrm{b}}$ \\
$\mathbf{2 a}$ & $0.30 \pm 0.03^{\mathrm{c}}$ & $0.43 \pm 0.04$ & $0.56 \pm 0.02^{\mathrm{bc}}$ & $0.73 \pm 0.021^{\mathrm{b}}$ \\
$\mathbf{2 b}$ & $0.28^{\mathrm{b}} \pm 0.02^{\mathrm{c}}$ & $0.41 \pm 0.02$ & $0.41 \pm 0.02^{\mathrm{bc}}$ & $0.13 \pm 0.02^{\mathrm{bc}}$ \\
$\mathbf{3 a}$ & $0.29^{\mathrm{b}} \pm 0.02^{\mathrm{c}}$ & $0.39 \pm 0.02$ & $0.39 \pm 0.02^{\mathrm{bc}}$ & $0.14 \pm 0.02^{\mathrm{bc}}$ \\
$\mathbf{3 b}$ & $0.37^{\mathrm{a}} \pm 0.03^{\mathrm{b}}$ & $0.74 \pm 0.03$ & $0.68 \pm 0.02^{\mathrm{b}}$ & $0.13 \pm 0.02^{\mathrm{bc}}$ \\
$\mathbf{4 a}$ & $0.37^{\mathrm{a}} \pm 0.03^{\mathrm{b}}$ & $0.74 \pm 0.03$ & $0.61 \pm 0.03^{\mathrm{b}}$ & $0.12 \pm 0.02^{\mathrm{bc}}$ \\
$\mathbf{4 b}$ & $0.15^{\mathrm{b}} \pm 0.02^{\mathrm{c}}$ & $0.34 \pm 0.03$ & $0.38 \pm 0.01^{\mathrm{c}}$ & $0.29 \pm 0.04^{\mathrm{c}}$ \\
$\mathbf{5 a}$ & $0.37^{\mathrm{a}} \pm 0.03^{\mathrm{b}}$ & $0.74 \pm 0.03$ & $0.61 \pm 0.02^{\mathrm{b}}$ & $0.30 \pm 0.05^{\mathrm{c}}$ \\
$\mathbf{5 b}$ & $0.22^{\mathrm{b}} \pm 0.02^{\mathrm{c}}$ & $0.31 \pm 0.04$ & $0.41 \pm 0.03^{\mathrm{b}}$ & $0.22 \pm 0.06^{\mathrm{b}}$ \\
$\mathbf{6 a}$ & $0.34^{\mathrm{a}} \pm 0.03^{\mathrm{b}}$ & $0.39 \pm 0.04$ & $0.54 \pm 0.03^{\mathrm{b}}$ & $0.42 \pm 0.02^{\mathrm{b}}$ \\
$\mathbf{6 b}$ & $0.35^{\mathrm{a}} \pm 0.02^{\mathrm{b}}$ & $0.42 \pm 0.03$ & $0.45 \pm 0.02^{\mathrm{b}}$ & $0.22 \pm 0.02^{\mathrm{b}}$ \\
$\mathbf{7 a}$ & $0.36^{\mathrm{a}} \pm 0.02^{\mathrm{b}}$ & $0.51 \pm 0.05$ & $0.48 \pm 0.02^{\mathrm{b}}$ & $0.32 \pm 0.02^{\mathrm{b}}$ \\
$\mathbf{7 b}$ & $0.32^{\mathrm{a}} \pm 0.04^{\mathrm{b}}$ & $0.52 \pm 0.04$ & $0.56 \pm 0.02^{\mathrm{b}}$ & $0.23 \pm 0.02^{\mathrm{b}}$ \\
$\mathbf{8 a}$ & $0.32 \pm 0.05^{\mathrm{b}, \mathrm{c}}$ & $0.42 \pm 0.03$ & $0.43 \pm 0.03^{\mathrm{b}}$ & $0.17 \pm 0.02^{\mathrm{b}}$ \\
$\mathbf{8 b}$ & $0.32 \pm 0.03^{\mathrm{b}, \mathrm{c}}$ & $0.39 \pm 0.02$ & $0.42 \pm 0.02^{\mathrm{b}}$ & $0.25 \pm 0.03^{\mathrm{b}}$ \\
$\mathbf{9 a}$ & $0.32 \pm 0.02^{\mathrm{b}, \mathrm{c}}$ & $0.56 \pm 0.02$ & $0.52 \pm 0.02^{\mathrm{b}}$ & $0.21 \pm 0.02^{\mathrm{b}}$ \\
$\mathbf{9 b}$ & $0.32 \pm 0.01^{\mathrm{b}, \mathrm{c}}$ & $0.47 \pm 0.01$ & $0.41 \pm 0.02^{\mathrm{b}}$ & $0.22 \pm 0.02^{\mathrm{b}}$ \\
$\mathbf{1 0 a}$ & $0.22 \pm 0.01^{\mathrm{b}, \mathrm{c}}$ & $0.46 \pm 0.01$ & $0.41 \pm 0.02^{\mathrm{b}}$ & $0.22 \pm 0.02^{\mathrm{b}}$ \\
$\mathbf{1 0 b}$ & $0.22 \pm 0.02^{\mathrm{b}, \mathrm{c}}$ & $0.42 \pm 0.02$ & $0.52 \pm 0.02^{\mathrm{b}}$ & $0.21 \pm 0.02^{\mathrm{b}}$ \\
$\mathbf{1 1 a}$ & $0.24 \pm 0.02^{\mathrm{b}, \mathrm{c}}$ & $0.43 \pm 0.01$ & $0.54 \pm 0.02^{\mathrm{b}}$ & $0.22 \pm 0.02^{\mathrm{b}}$ \\
$\mathbf{1 1 b}$ & $0.52 \pm 0.02^{\mathrm{b}, \mathrm{c}}$ & $0.48 \pm 0.013$ & $0.56 \pm 0.02^{\mathrm{b}}$ & $0.22 \pm 0.02^{\mathrm{b}}$ \\
\hline
\end{tabular}

a Mean \pm SE $(n=8)$.

b Significantly different from normal control value ( $p \leq 0.05)$.

c Significantly different from testosterone value $(p \leq 0.05)$.

Groups of prepubertal albino rats $(n=8) 21$ days old, were kept on a constant diet and tap water. Each animal was given daily a subcutaneous injection of the tested compound as well as testosterone as reference standard at a dose of $0.1 \mathrm{mg} \mathrm{kg}^{-1}$ for seven days. On day eight (22-26 hours after the last injection), the animals were killed, dissection of the levator ani muscle, the ventral prostate gland, testis, seminal vesicles, vas deference and epididymis were carried out and were weighed.

The ratio of the mass gain of the levator ani-muscle to the mass gain of the ventral prostate gland was calculated where the mass gain of the levator ani muscle indicates 
N. A. Abd El-Latif et al.: New steroidal derivatives synthesized using 33-hydroxyandrosten-17-one as starting material, Acta Pharm. 58 (2008) $43-59$.

the anabolic effect and the mass gain of the ventral prostate gland shows the androgenic effect of the tested compounds.

\section{RESULTS AND DISCUSSION}

In the present work, we utilized Strechker synthesis to introduce the $17 \beta$-cyano$-17 \alpha$-substituted amino group into synthesized compounds, because the cyano group provides a wide variety of biological activities. Amino group serves to form water-soluble salts that increase the pharmacokinetics and pharmacodynamics of steroids such as Stanazolole ${ }^{\circledR}$ due to its increased absorption and distribution in plasma protein.

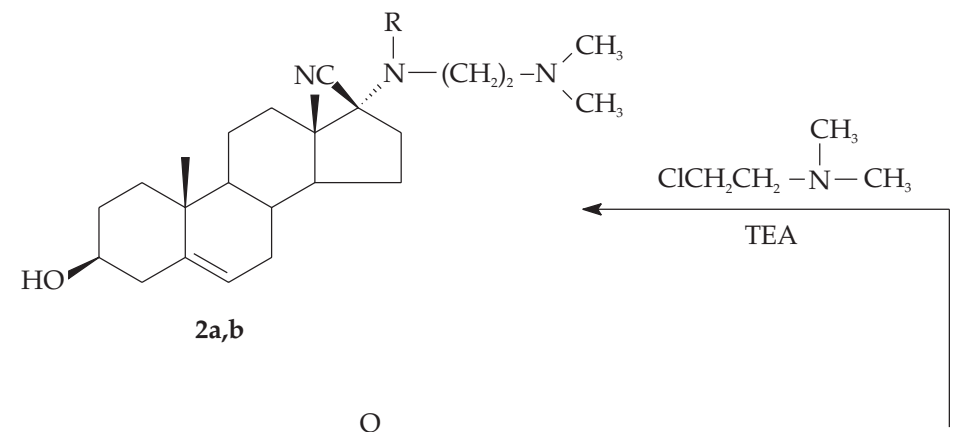

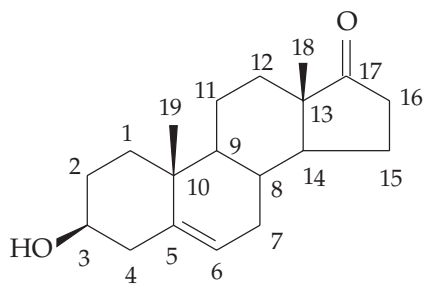<smiles>C[C@@]12CCC(O)CC1=CCC1C2CC[C@@]2(C)C1CCC2(N)N</smiles>

Dehydroepiandrosterone

$$
1 \mathrm{a}, \mathrm{b}
$$

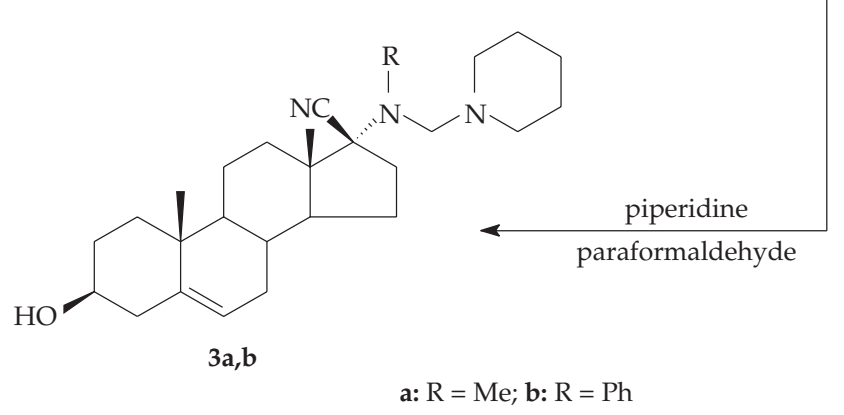

Scheme 1 
Compounds $\mathbf{1} \mathbf{a}, \mathbf{b}$ were obtained by reacting dehydroepiandrosterone with primary amines, namely, methylamine or aniline in the presence of potassium cyanide. Reaction of compounds $\mathbf{1} \mathbf{a}, \mathbf{b}$ with dimethylamino-2-chloroethane in the presence of triethylamine as catalyst yielded $N, N$-dimethylethane derivatives $\mathbf{2} \mathbf{a}, \mathbf{b}$ while the reaction of $\mathbf{1} \mathbf{a}, \mathbf{b}$ with piperidine in the presence of paraformaldehyde gave the piperidyl derivatives $\mathbf{3} \mathbf{a}, \mathbf{b}$. The IR spectra of 1-3 showed the absence of bands corresponding to $v(\mathrm{C}=\mathrm{O})$ for dehydroepiandrosterone and the presence of a band at $2224-2227 \mathrm{~cm}^{-1}$ corresponding to $v(\mathrm{C} \equiv \mathrm{N})$, and a broad band at 3448-3396 $\mathrm{cm}^{-1}$ corresponding to $v(\mathrm{NH})$ for dehydroepiandrosterone. The syntheses of compounds 1-3 are outlined in Scheme 1.

Acylation of the resulting compounds $\mathbf{1} \mathbf{a}, \mathbf{b}$ with acetylchloride or trifluoroacetic anhydride in boiling toluene gave the corresponding diprotected derivatives $\mathbf{4 a}, \mathbf{b}$ and $\mathbf{5} \mathbf{a}, \mathbf{b}$, respectively. Also, catalytic hydrogenation of $\mathbf{1} \mathbf{a}, \mathbf{b}$ with palladium charcoal $(\mathrm{Pd} /$<smiles></smiles>

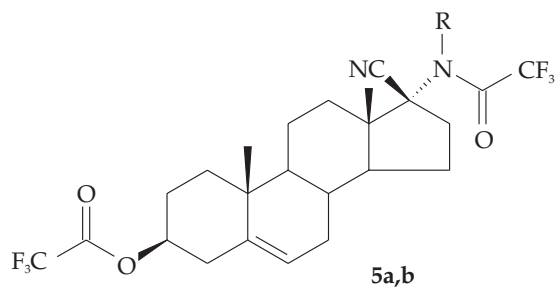

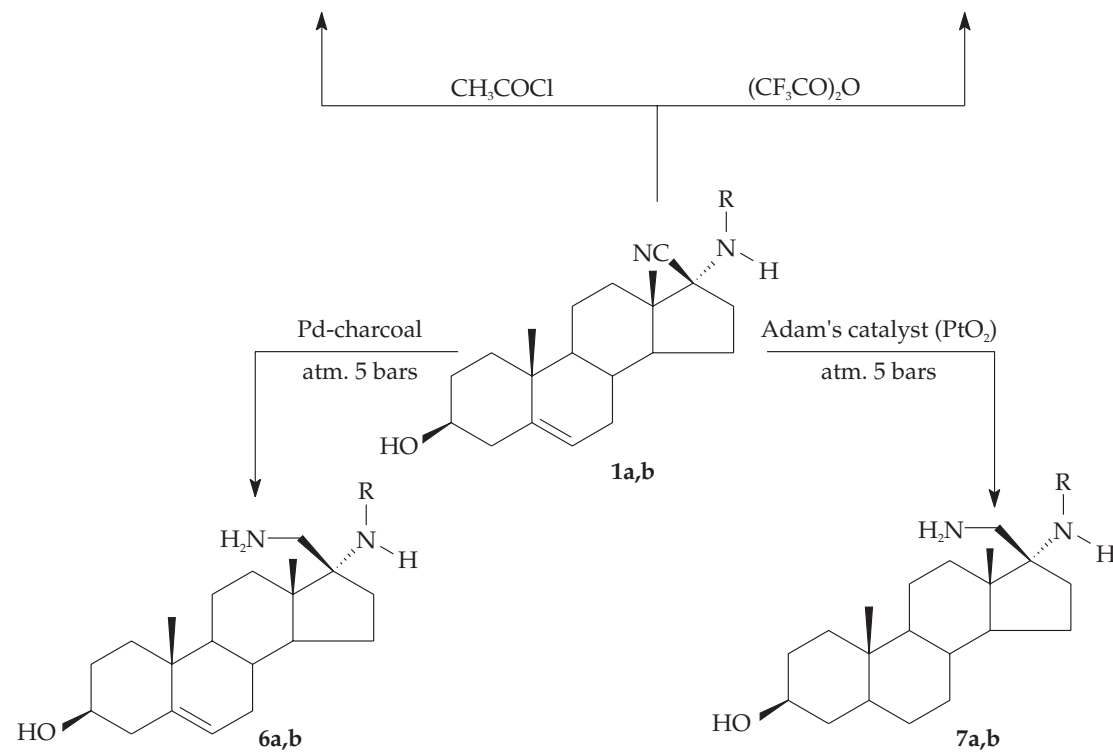

a: $\mathrm{R}=\mathrm{Me} ; \mathrm{b}: \mathrm{R}=\mathrm{Ph}$

Scheme 2 
charcoal) afforded the corresponding primary amines $6 \mathbf{a}, \mathbf{b}$ without affecting the $\Delta^{5}$-ene double bond, whereas with Adam's catalyst $\left(\mathrm{PtO}_{2}\right)$ it afforded the corresponding primary amines $7 \mathbf{a}, \mathbf{b}$ with hydrogenated $\Delta^{5}$-ene double bond. The IR spectra of 4 and $\mathbf{5}$ showed the absence of bands $v(\mathrm{OH})$ at $3448-3378 \mathrm{~cm}^{-1}$ for the starting compound and the presence of bands corresponding to $v(\mathrm{C}=\mathrm{O})$ for 4 and 5 . Also, the IR spectra of 6 and 7 showed the absence of bands $v(\mathrm{CN})$ at $2224 \mathrm{~cm}^{-1}$ for the starting compound and the presence of bands corresponding to $v\left(\mathrm{NH}_{2}\right)$ for 6 and 7 . The syntheses of compounds 4-7 are outlined in Scheme 2.

Hydrolysis of compounds $\mathbf{1} \mathbf{a}, \mathbf{b}$ with sulphuric acid $\left(\mathrm{H}_{2} \mathrm{SO}_{4}, 0.5 \mathrm{~mol} \mathrm{~L}-1\right)$ or alcoholic sodium hydroxide (10\%) gave the corresponding amide derivatives $\mathbf{8} \mathbf{a}, \mathbf{b}$ and the carboxylic acids $\mathbf{9} \mathbf{a}, \mathbf{b}$, respectively. On the other hand, oxidation of $\mathbf{1} \mathbf{a}, \mathbf{b}$ with Killian reagent (chromic acid, as oxidizing agent) afforded the corresponding 3-oxo-analogues $\mathbf{1 0 a , b}$ with hydrolyzed cyano group to carboxylic group. Condensation of compounds $\mathbf{1 a}, \mathbf{b}$ wi-

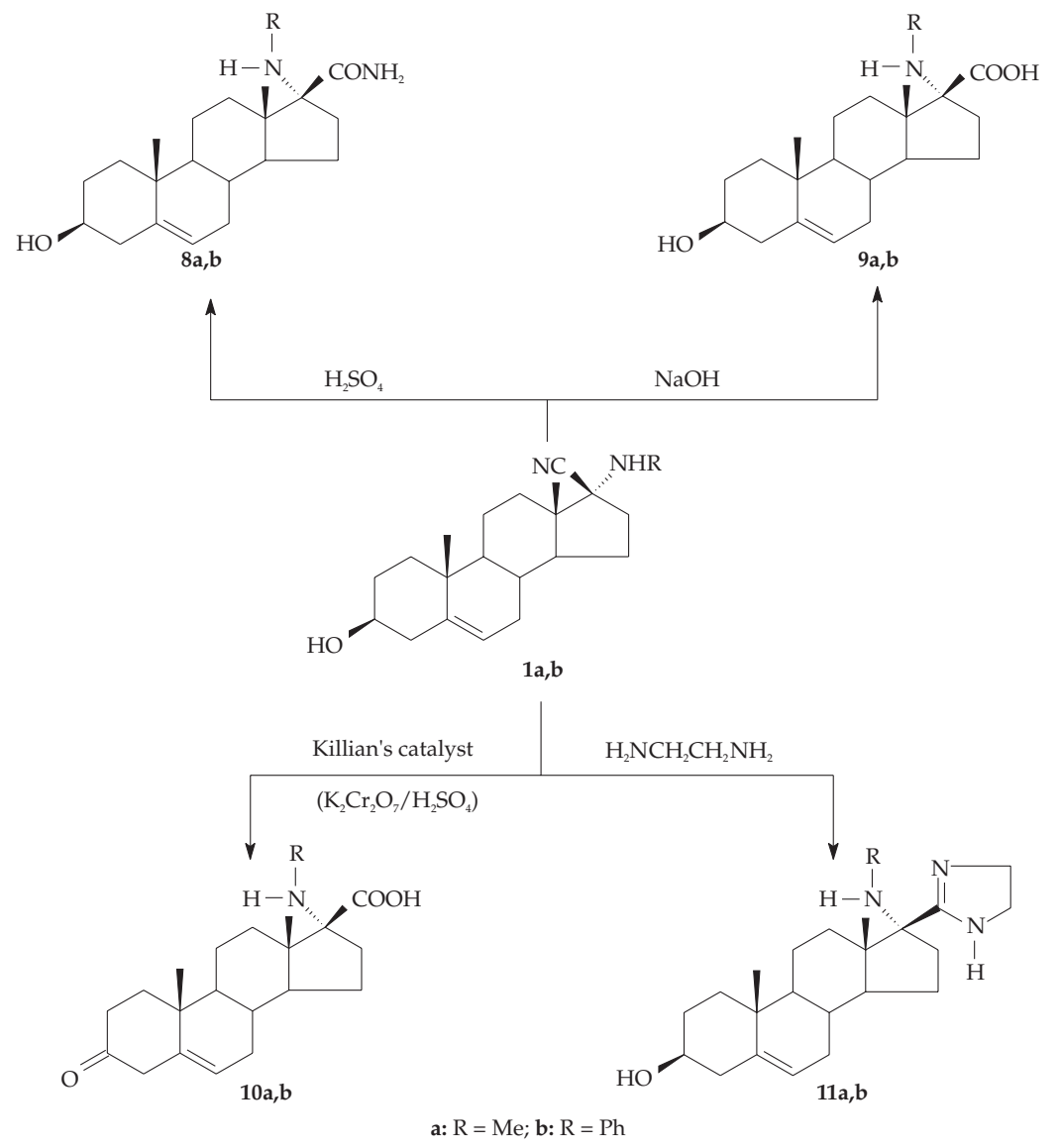

Scheme 3 
th 1,2-diaminoethane gave the corresponding imidazole derivatives $\mathbf{1 1 a}, \mathbf{b}$. The IR spectra of 8-11 showed the absence of bands corresponding to $v(\mathrm{C} \equiv \mathrm{N})$ for $\mathbf{1}$ and the presence of bands corresponding to $v(\mathrm{C}=\mathrm{O})$ for compounds 8-10 and $v(\mathrm{NH})$ for 11. The syntheses of compounds 8-11 are outlined in Scheme 3.

\section{Acute toxicity}

Initially, acute toxicity of the synthesized compounds was assayed by determining their $L D_{50}$. Interestingly, most compounds were less toxic than the reference drug, except compounds $\mathbf{1} \mathbf{b}, \mathbf{4} \mathbf{b}, \mathbf{6} \mathbf{a}, \mathbf{7} \mathbf{b}$ and $\mathbf{8} \mathbf{b}$ (Table III). The $L D_{50}$ (rats) was determined by injecting different increasing doses and calculating the dose that killed $50 \%$ of the animals.

\section{Androgenic-anabolic activity}

The newly synthesized compounds were then pharmacologically screened for their androgenic-anabolic potency on male albino rats.

From Tables IV and V, the ratio of the mass gained by the levator ani-muscle to the mass gained by the prostate gland was calculated, where the former indicates the anabolic activity and the latter shows the androgenic effect of the tested compounds. It was revealed that all the tested compounds have significant androgenic as well as anabolic effects. Regarding the androgenic activity indicated by the mass gained by the prostate gland, compounds $\mathbf{3 a}, \mathbf{6 b - 1 1} \mathbf{b}$ showed high androgenic activity.

Compounds $\mathbf{1 a}, \mathbf{1 b}, \mathbf{2 a}, \mathbf{2 b}, \mathbf{3 b}, \mathbf{4 a}, \mathbf{4 b}, \mathbf{5 a}$ and $\mathbf{5 b}$ showed potent anabolic activities where the ratio of mass gained by the levator ani-muscle (anabolic) to the mass gained by the ventral prostate were 2.20, 1.90, 1.82, 1.28, 1.09, 1.08, 1.68, 2.30 and 1.06 (Table IV).

It is evident from Table $\mathrm{V}$ that compounds $\mathbf{3 a}, \mathbf{6 b}-\mathbf{1 1} \mathbf{b}$ significantly increase the mass of male sex organs. Compound $\mathbf{5 a}$ showed high anabolic activity, increasing the mass of both levator-ani muscle and prostate gland in a statistically significant manner. It increased the mass of the testicles but in a non-significant manner.

\section{Structure activity relationship (SAR)}

The presence of some groups (hydrogen bond donors) is beneficial for androgenic activities. The activity was found to decrease in the following order: $6 a, 6 b, 8 a, 7 b, 11 b$ and $\mathbf{7 a}$. It was found that the anabolic activity was decreasing in the order: $\mathbf{5 a}, \mathbf{1 a}, \mathbf{1} \mathbf{b}, \mathbf{4} \mathbf{b}$, $2 a, 3 b, 4 a$ and $5 b$, indicating that the presence of hydrogen bond acceptor is an important beneficial factor for anabolic activity.

\section{CONCLUSIONS}

Twenty-two steroidal derivatives were synthesized and tested for their androgenic-anabolic activities. The substituted steroidal structure containing 5-ene double bonds was found essential for androgenic-anabolic activity. A future work will involve the design of steroidal molecules of such features. 


\section{REFERENCES}

1. A. E. Amr and M. M. Abdalla, Synthesis and anti-inflammatory activities of new cyanopyrane derivatives fused with steroidal nuclei, Arch. Pharm. Chem. Life Sci. 339 (2006) 88-95; DOI: 10. 1002/ardp.2005000209.

2. A. E. Amr, N. A. Abdel-Latif and M. M. Abdalla, Synthesis and antiandrogenic activity of some new 3-substituted androstano-[17,16-c]-5'-aryl-pyrazoline and their derivatives, Bioorg. Med. Chem. 14 (2006) 373-384; DOI: 10.1016/j.bmc.2005.08.024.

3. A. E. Amr, M. I. Hegab, A. A. Ibrahim and M. M. Abdulla, Synthesis and reactions of some fused oxazinone, pyrimidinone, thiopyrimidnone and triazinone derivatives with thiophene ring as analgesic, anticonvulsant and antiparkinsonian agents, Monatsh. Chem. 134 (2003) 1395-1409; DOI: $10.1007 /$ s00706-003-0051/-z.

4. M. H. Abou-Ghalia and A. E. Amr, Synthesis and investigation of a new cyclo(N ${ }^{\alpha}$-di-picolinoyl)pentapeptide of a breast and CNS cytotoxic activity and an ionophoric specificity, Amino Acids 26 (2004) 283-289; DOI: 10.1007/s00726-003-0042-8.

5. M. F. Brana, J. M. Castellano, M. Mpran, M. J. Perez de Vega, X. D. Gian, C. A. Romerdahl, G. Keihauer, Bis-naphthalimides. 2. Synthesis and biological activity of 5,6-acenaphthalimidoalkyl-1,8-naphthalimidoalkyl amines, Eur. J. Med. Chem. 30 (1995) 235-239; DOI: 10.1016/02235234(96)88230-4.

6. A. E. Amr, A. M. Mohamed, S. F. Mohamed, N. A. Abdel-Hafez and A. G. Hammam, Synthesis and anticancer activities of new pyridine, pyrane and pyrimidine derivatives fused with nitrobenzosubetrone moiety, Bioorg. Med. Chem. 14 (2006) 5481-5488; DOI: 10.1016/j.bmc.2006.04. 045 .

7. A. E. Amr, A. M. Mohamed and A. A. Ibrahim, Synthesis of some new chiral tricyclic and macrocyclic pyridine derivatives as antimicrobial agents, Z. Naturforsch. 58b (2003) 861-868; DOI: 932-0776/03/0900-0861.

8. A. E. Amr, O. I. Abdel-Salam, A. Attia and I. Stibor, Synthesis of new potential bis-intercallators based on chiral pyridine-2,6-dicarboxamides, Collect. Czech. Chem. Commun. 64 (1999) 288-298 DOI: $10.1135 /$ ccc19990288.

9. A. Attia, O. I. Abdel-Salam, A. E. Amr, I. Stibor and M. Budesinsky, Synthesis and antimicrobial activity of some new chiral bridged macrocyclic pyridines, Egypt. J. Chem. 43 (2000) 187-201; DOI: $10.1007 / 500726-003-0042-8$

10. A. E. Amr, Synthesis of some new linear and chiral macrocyclic pyridine carbazides as analgesic and anticonvulsant agents, Z. Naturforsch. 60b (2005) 990-998; DOI: 0932-0776/05/0900-0990.

11. M. H. Abou-Ghalia, A. E. Amr and M. M. Abdalah, Synthesis of some new ( $\mathrm{N}^{\alpha}$-dipicolinoyl)bis-L-leucyl-DL-norvalyl linear tetra and cyclic octa bridged peptides as new antiinflammatory agents, Z. Naturforsch. 58b (2003) 903-910; DOI: 0932-0776/03/0900-0903.

12. A. E. Amr and M. M. Abdulla, Synthesis and pharmacological screening of some new pyrimidine and cyclohexenone fused steroidal derivatives, Indian J. Heterocycl. Chem. 12 (2002) 129-134.

13. J. C. Jung, E. B. Watkins and M. A. Avery, Synthesis and cyclization reaction of pyrazolin-5-one derivatives, Heterocycles 65 (2005) 77-94.

14. A. E. Amr, H. S. Hasanien and M. M. Abdalla, Synthesis and reactions of some new substituted pyridine and pyrimidine derivatives as analgesic, anticonvulsant and antiparkinsonian agents, Arch. Pharm. Chem. Life Sci. 338 (2005) 433-440; DOI: 10.1002/ardp.200500982.

15. E. Bansal, V. K. Srivastava and A. Kumar, Synthesis and anti-inflammatory activity of 1-acetyl-5-substituted diaryl-3-( $\beta$-aminoacyl)-2-pyrazolines and $\beta$-(substituted-diaminoethyl)-amidonaphthalenes, Eur. J. Med. Chem. 36 (2001) 81-92; DOI: 10.1016/SO223-5234(00)01179-X. 
16. J. H. Ahn, H. M. Kim, S. H. Jung, S. K. Kang, K. R. Kim, S. D. Rhee, S. D. Yong, H. G. Cheon, S. S. Kim, Synthesis and DP-IV inhibition of cyanopyrazoline derivatives as potent anti-diabetic agents, Bioorg. Med. Chem. Lett. 14 (2004) 4461-4465; DOI: 10.1016/j.bmcl.2004.06.046.

17. A. E. Amr, Synthesis of some heterocyclic compounds as potential antimicrobial agents using 2,6-diacetylpyridine as synthon, Indian J. Heterocycl. Chem. 10 (2000) 49-58.

18. A. G. Hammam, A. F. M. Fahmy, A. E. Amr and A. M. Mohamed, Synthesis of novel tricyclic heterocyclic compounds as potential anticancer agents using chromanone and thiochromanone as synthons, Indian J. Chem. Sect. B. 42B (2003) 1985-1993.

19. A. G. Hammam, N. A. Abdel Hafez, W. H. Midura and M. Mikolajczyk, Chemistry of seven membered heterocycles. VI. Synthesis of novel bicyclic heterocyclic compounds as potential anticancer and anti-HIV agents, Z. Naturforsch. 55b (2000) 417-424.

20. S. S. M. Hassan, M. H. Abou-Ghalia, A. E. Amr and A. H. K. Mohamed, New lead (II) selective membrane potentiometric sensors based on chiral 2,6-bis-pyridine-carboxamide derivatives, Talanta 60 (2003) 81-91; DOI: 10.1016/S0039-9140(03)00038-9.

21. S. S. M. Hassan, M. H. Abou-Ghalia, A. E. Amr and A. H. K. Mohamed, Novel thiocyanate-selective membrane sensors based on di-, tetra-, and hexa-imidepyridine ionophores, Anal. Chim. Acta 482 (2003) 9-18; DOI: 10.1016/S003-2670(3)00172-7.

22. K. F. Austen and W. E. Brocklehurst, Anaphylaxis in chopped guinea pig lung: I. Effect of peptidase substrates and inhibitors, J. Exp. Med. 113 (1961) 521-539.

23. L. G. Hershberger, E. G. Shipley and R. K. Meyer, Myotrophic activity of 19-nortestosterone and other steroids determined by modified levator ani muscle method, Proc. Soc. Exp. Biol. Med. 83 (1953) $175-180$.

24. W. Kuhnz and S. Beier, Comparative progestational and androgenic activity of norgestimate and levonorgestrel in the rat, Contraception 49 (1994) 275-289; DOI: 10.016/0010-7824(94)90045-0.

\section{$S A \check{Z} E T A K$}

\section{Novi steroidni derivati sintetizirani iz $3 \beta$-hidroksiandrosten-17-ona}

NEHAD A. ABD EL-LATIF, MOHAMED M. ABDULLA i ABD EL-GALIL E. AMR

U radu je opisana sinteza novih steroidnih derivata 1-11 koristeći 3ß-hidroksiandrosten-17-on (dehidroepiandrosteron) kao početnu supstanciju. Androgeno-anaboličko djelovanje tih spojeva uspoređivano je s djelovanjem testosterona kao pozitivnom kontrolom. Navode se detaljni sintetski postupci, spektroskopska karakterizacija i podaci o toksičnosti $\left(L D_{50}\right)$.

Ključne riječi: dehidroepiandrosteron, steroidni derivati, androgeno-anabolički steroidi

Natural Compounds Department, National Research Center, Cairo, Dokki, Egypt

Research Units, Hi-Care Pharmaceutical Co., Cairo, Egypt

Applied Organic Chemistry Department, National Research Center, Cairo, Dokki, Egypt 